\title{
A Generalised Asperity-Based Friction Model
}

\author{
Kris De Moerlooze $\cdot$ Farid Al-Bender • \\ Hendrik Van Brussel
}

Received: 19 April 2010/ Accepted: 11 June 2010/Published online: 29 June 2010

(C) Springer Science+Business Media, LLC 2010

\begin{abstract}
Despite considerable research effort, the use of physics-based modelling to predict frictional behaviour is still a debatable question in modern tribological research. This article presents a dry-friction model, based on physical phenomena such as adhesion, elastic-plastic contact and deformation. This contribution offers a means to simulate all kinds of frictional behaviour that is observed in experimental research. The contact of two bodies through their surfaces is transformed into the contact of a body that is provided with asperities and containing material and geometrical information of both of the mating surfaces, and a counter profile, holding solely geometrical information. The local adhesion between the asperity tips and the counter profile, together with the elastic-plastic behaviour of the asperities themselves, form the basis for this model. The simulation results show qualitatively good agreement with experimental study. Friction and contact phenomena such as normal creep, increasing static coefficient of friction with increasing dwell time, pre-sliding hysteresis with nonlocal memory, Stribeck and viscous effect, frictional lag, stick-slip and dynamical oscillations are revealed by this model. Furthermore, future improvement and refinement of the model is possible (and ongoing) so as to incorporate lubrication and asperity wear.
\end{abstract}

Keywords Friction mechanisms · Dynamic modelling

\section{List of Symbols}

$\delta_{j} \quad$ Spring deformation of the $j$-th element

$\delta_{\mathrm{n}} \quad$ Normal interference depth

K. De Moerlooze $(\varangle) \cdot$ F. Al-Bender · H. Van Brussel Mechanical Engineering Department, Division PMA, Katholieke Universiteit Leuven, Celestijnenlaan 300B, 3001 Heverlee, Belgium

e-mail: Kris.DeMoerlooze@mech.kuleuven.be
$\Delta_{j} \quad$ Maximum spring deformation of the $j$-th Maxwell-Slip element

$\zeta \quad$ Main damping ratio

$\zeta_{\mathrm{T} i} \quad$ Tangential damping ratio of the $i$-th asperity

$\zeta_{\mathrm{N} i} \quad$ Normal damping ratio of the $i$-th asperity

$\lambda \quad$ Mean counter profile wavelength

$\mu \quad$ Coefficient of friction

$\mu_{\mathrm{s}} \quad$ Static coefficient of friction

$\sigma_{\mathrm{N} i}, \sigma_{\mathrm{T} i} \quad$ Yielding force

$\tau \quad$ Non-dimensional time

$\omega_{\mathrm{N}} \quad$ Main eigenfrequency of the system

$\omega_{\mathrm{nT} i} \quad$ Tangential eigenfrequency of the $i$-th asperity

$\omega_{\mathrm{nN} i} \quad$ Normal eigenfrequency of the $i$-th asperity

$\omega_{\mathrm{nP} i} \quad$ Eigenfrequency of counter profile of the $i$-th asperity

$A \quad$ Amplitude of the applied excitation

$c_{\mathrm{T}, i}, c_{\mathrm{N}, i}$ Dimensional tangential and normal damping of the $i$-th asperity

$C_{\mathrm{T}} \quad$ Dimensional main tangential damping

C Damping matrix

$d x_{\mathrm{T}, i} \quad$ Horizontal asperity distribution

$f_{\mathrm{t}} \quad$ Frequency of the applied excitation

$F_{\text {fric }} \quad$ Non-dimensional global friction force

$F_{\text {norm }} \quad$ Non-dimensional global normal contact force

$F_{\text {n }} \quad$ Local normal contact force

$F_{\text {adh }, i} \quad$ Adhesion force of the $i$-th asperity

F $\quad$ Force vector

$k_{\mathrm{el}} \quad$ Elastic spring stiffness

$k_{\mathrm{pl}} \quad$ Plastic spring stiffness

$k_{\mathrm{p}, i} \quad$ Profile stiffness of the $i$-th asperity

$k_{\mathrm{T}, i}, k_{\mathrm{N}, i}$ Dimensional tangential and normal stiffness of the $i$-th asperity

$K_{\mathrm{T}} \quad$ Dimensional main tangential stiffness

K Stiffness matrix

$l_{i} \quad$ Dimensional asperity length 


$\begin{array}{ll}L_{i} & \text { Non-dimensional asperity length } \\ m_{i} & \text { Dimensional mass of the } i \text {-th asperity } \\ M & \text { Dimensional main mass } \\ \mathbf{M} & \text { Mass matrix } \\ N & \text { Number of asperities } \\ q & \text { State vector } \\ t & \text { Dimensional time } \\ x, y & \text { Dimensional position } \\ x_{i}, y_{i} & \text { Dimensional asperity mass position of the } i \text {-th } \\ & \text { asperity } \\ x_{\mathrm{m}}, y_{\mathrm{m}} & \text { Dimensional position of the free end of the main } \\ & \text { spring } \\ x_{\mathrm{t}}, y_{\mathrm{t}} & \text { Dimensional main mass position } \\ X, Y & \text { Non-dimensional position } \\ i & \text { Dimensional time derivation } \\ & \text { Non-dimensional time derivation }\end{array}$

\section{Introduction}

The innovative study of Leonardo da Vinci [1], Guillaume Amontons [2], Leonard Euler [3] and Charles Augustin de Coulomb [4], concentrated on investigating the complex mechanics of the relative motion between contacting bodies. However, over the last 500 years, the main research effort was focused on the mechanisms giving rise to the frictional resistance, in particular adhesion and deformation [5], while friction as a dynamical process evolving in the contact was often overlooked. The profound understanding of this dynamical behaviour is of crucial importance in diverse applications, ranging from high precision machine elements over biomedical and automotive applications to earthquake engineering. The exigency for a model capable of simulating the dynamical behaviour is evident for studying stick-slip motion, where the unstable motion originates from the interaction of the frictional dynamics and the rest of the mechanical system. In order to study this phenomenon on a theoretical basis, a realistic model is needed for the frictional behaviour in the contact of the mating surfaces.

Despite numerous attempts, the modelling of dry friction between solids remains a pertinent issue in modern tribological research. The reason may be found in the complexity of the processes taking place in any tribological contact, on different space and time scales, including elastic and plastic deformation of bodies, material properties, lubrication, thermal effects, humidity, fracture and wear, reintegration of particles into the surface, chemical reactions and mechanical interactions $[6,7,8]$.

Another difficult issue which Popov and Psakhie [6] mention, is the multi-scale nature of friction. Microscopic scales affect the friction properties, such as a thin layer of impurities on a metal surface drastically change the friction properties [9]. Conversely, the time and space scales of contact mechanics can differ by many orders of magnitude [10].

The asperity-based modelling has a long history. One of the earliest studies on the modelling of systems at the asperity level, is probably the study of Abbott and Firestone [11], where the contact was simulated with a network of spheres, which are truncated by the indentation with a rigid flat surface. More than three decades later, Kragelsky [12], considers a rough surface as a composition of elastic rods with different lengths, fixed from one end to a rigid surface. Based on a statistical approach, the global coefficient of friction is function of the deformation of the material, the geometrical properties and the local friction force, of which the latter is considered to be pressure dependent. This model shows an increasing coefficient of friction with decreasing surface roughness, in accord with experimental results.

Greenwood and Williamson [13] also base their study on multi-asperity behaviour, considering the asperity tips as hemispheres with a constant radius of curvature. The contact between two rough surfaces is transformed into the contact of an equivalent rough surface and a rigid flat surface. Whitehouse and Archard [14] extend the model of Greenwood and Williamson by studying a random variation of radius of curvature of the asperity tips. Ogilvy [15] develops a numerical strategy to simulate the frictional behaviour of rough surfaces, using Hertzian contact analysis [16] and simple plasticity theory. The coefficient of friction is calculated on both the micro scale and the macro scale. On the micro scale, the adhesive forces required to break all junctions are summed up, while in the macroscopic approach, the friction force is related to the total area of true contact. Agreement of the numerical results with experimental study is found in the contact of substrates with a $\mathrm{MoS}_{2}$ coating.

Ford [17] bases his multi-asperity model on a combination of the models of Tabor [18] and Greenwood and Williamson [13], to study the contact angle and its distribution for pure elastic and pure plastic indentation. The numerical code utilised, therefore, is based on the Ogilvy model [15], where the asperities are no longer considered as hemispheres of equal radius.

The CEB model of Chang, Etsion and Bogy [19] incorporates the elastic-plastic behaviour of the contacting asperities.

Tworzydlo et al. [21] present a constitutive model of frictional interfaces, with the aim of providing the link between micro scale effects at the asperity contacts and macroscopic phenomena of the contacting materials. They make use of a special finite element method, combined 
with empirical findings for the local friction force and a statistical homogenisation technique.

Karpenko and Akay [22] describe a computational method for the simulation of the friction force in steady sliding of three-dimensional rough surfaces. They assume the friction force to originate from the elastic asperity deformation and the shearing due to the adhesion occurring the junctions. Based on the local friction dependency on the normal pressure and the arbitrary orientation and contact of asperities, they develop an iterative procedure utilising a successive grid refinement.

Several research groups use a FEM-based approach to model the contact between a rigid flat surface and a rough surface, where the asperities are modelled as hemispheres. Jackson and Green [23] develop empirical formulas for the elastic-plastic contact in this manner. Quicksall et al. [24] extend this study for a set of materials and compare the results obtained with the Jackson and Green model.

None of the previously presented modelling strategies are, however, capable of simulating dynamic frictional phenomena such as the Stribeck effect, viscous sliding and friction lag. In order to manage this, a completely different modelling approach is necessary.

During the last two decades, another category of friction models were being developed, namely heuristic models for friction force dynamics, intended to be used for control purposes [25]. Such models are not concerned with actual contact mechanics, but are parametric mathematical formulations that can be used for fitting experimentally obtained friction behaviour. A special case of these is the Generic model proposed in [26], which uses phenomenological mechanisms to formulate the dynamics of asperity interaction.

The objective of this study is to formulate a model that combines the approaches and features of both categories of models reviewed above, so as to result in a most general and physically realistic representation of the contact and friction phenomenon.

This article presents a generic, wear-less dry friction model, which incorporates both static (deformation, adhesion) aspects and dynamic behaviour. With this model, one is able to simulate the dynamics of the interaction of a body comprising a mass and a set of asperities under normal loading and tangential excitation. Despite relatively simple assumptions, the model is able to simulate all experimentally observed frictional behaviours. The article is structured as follows. In Sect. 2, the ingredients of the model are discussed, leading to the renewed formulation of the generic model. This is followed in Sect. 3 by an enunciation of the numerical strategy and the utilised normalisation. Section 4 overviews the obtained results to show the relevance of this contribution. Finally, some appropriate conclusions are drawn.

\section{Model Description}

Geike and Popov [27] show that three-dimensional contacts have the same force-displacement law as a reduced onedimensional model. This grants one to reduce a threedimensional asperity based rough surface into a more manageable and computable system, in which each asperity is converted to a mass, connected via a normal and tangential spring, to the main mass. This reduction allows us to describe the dynamical frictional behaviour of rubbing surfaces, which is mandatory for an accurate modelling of the frictional behaviour of these contacts.

\subsection{The Contact Scenario}

The contact of two surfaces sliding over each other, can be represented by a mass with a flexible surface, containing all asperities, and a counter profile, determined by a certain geometrical form and a certain stiffness, which is much higher than the stiffness properties of the asperities. The two first bodies are consequently transformed to an upper body, containing all geometric and material information, and a counter profile, containing only geometrical information.

The top surface is represented by asperities, each of which has a certain mass, stiffness, damping and length which, combined with the shape of the counter surface, forms the transformation of the original local asperity contact. For the sake of simplicity, the asperity mass is considered as a point mass, i.e. without geometrical dimensions. This is illustrated in Fig. 1, where the varying radius of the asperity is a measure for the stochastic distribution of the asperity masses.

The main mass of the sliding body $M$ can be excited in different ways. One can either control its motion by a direct application of an external force or displacement, or realise the same through a parallel connection of a spring $K_{\mathrm{T}}$ and a damper $C_{\mathrm{T}}$ to the mass, while controlling the position of the other (free) end of the spring/damper combination.

An asperity is represented by its mass $m_{i}$, the normal and tangential stiffness $k_{\mathrm{N} i}, k_{\mathrm{T} i}$ and damping $c_{\mathrm{N} i}, c_{\mathrm{T} i}$, where $i$ stands for the $i$-th asperity of a set of size $N$. In that way, normal and tangential behaviour of the asperity are decoupled. The undeformed length of the asperity is characterised by its relaxation length $l_{i}$. This is presented in Fig. 1, where the rest length of asperity 1 is indicated by $l_{1}$.

In contact modelling, two contacting rough surfaces are often reduced to the contact of one surface, having the equivalent roughness of both original surfaces, and a perfectly flat surface (e.g. $[13,28])$. This reduction is plausible for stationary contact simulation, but fails when considering frictional dynamics in sliding contacts, since it misses the ability of asperity interlocking and an incorrect 
Fig. 1 The contact scenario

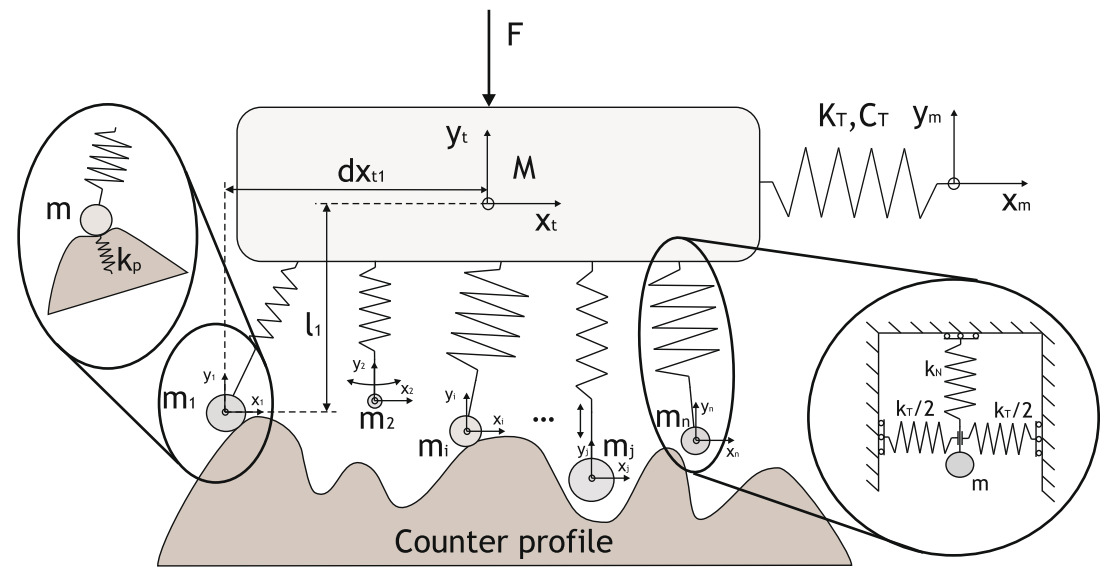

representation is given of the continuously changing relative topology [26]. Therefore, the asperity population, representing the top surface, is given a certain length distribution, reflecting the equivalent roughness of the original surfaces. On the other hand, the lower surface is proposed as a profile with constant amplitude, representing a measure for the overlap between asperities during contact, while the wavelength is chosen arbitrarily, from a given distribution. Various distributions for the asperity length are applicable, such as exponential and Gaussian distributions for the asperity heights, as Greenwood and Williamson [13] propose. Persson et al. [10] indicate the Gaussian nature of the height distribution of natural surfaces, such as surfaces prepared by fracture or sputtering, while polished rough surfaces show a non-symmetric height distribution. Andrews and Sehitoglu [29] propose a log-normal distribution for the asperity height, tip radius and horizontal position. However, Lampaert [25] shows that the type of distribution has only a minor influence on the hysteresis friction behaviour.

In conformity with the Greenwood-Williamson assumption [13], no interaction forces between asperities are modelled. Contact between upper surface and lower surface occurs when an asperity of the top surface penetrates (i.e. interferes with) the lower surface. A proper choice of the counter surface accelerates the calculation of the interference depth $\delta$. An extra stiffness $k_{\mathrm{p} i}$, the profile stiffness, affects the motion of the asperity mass $m_{i}$ in the normal direction of the counter profile with a force $F_{\mathrm{n}}=k_{\mathrm{p} i} \delta_{\mathrm{n}}$, where $\delta_{\mathrm{n}}$ is the normal interference. Besides the contact interaction of the asperity and the counter profile, an additional adhesion mechanism is modelled. The tangential adhesion law used by Al-Bender et al. [26] must be understood as a tangential surface force, resisting tangential motion. This adhesion law is depicted in the left panel of Fig. 2. The case of the more classical adhesion laws [20, 30, $31,32]$, where the asperity is subjected to an attractiverepulsive force in the normal direction towards the contacting surface, is depicted in the right panel of Fig. 2. In order to describe the force-separation relationship, the Dugdale approximation [33] is used, where the integral of the forceseparation curve is a measure for the work of adhesion. Further below, the results yielded by these two approaches are compared and discussed.
Fig. 2 Left panel the adhesion law in the tangential direction, opposite to the velocity of the asperity, right panel the normal adhesion law, an attractive force is exerted to the asperity when the adhesion zone is entered tangential adhesion

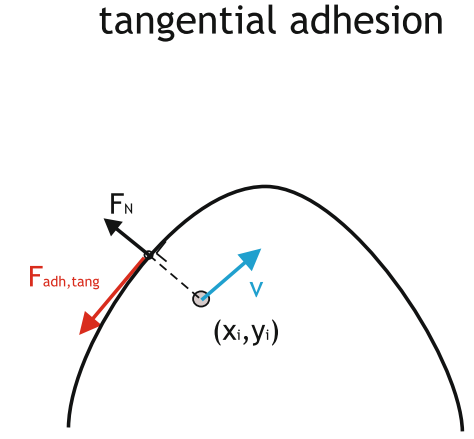

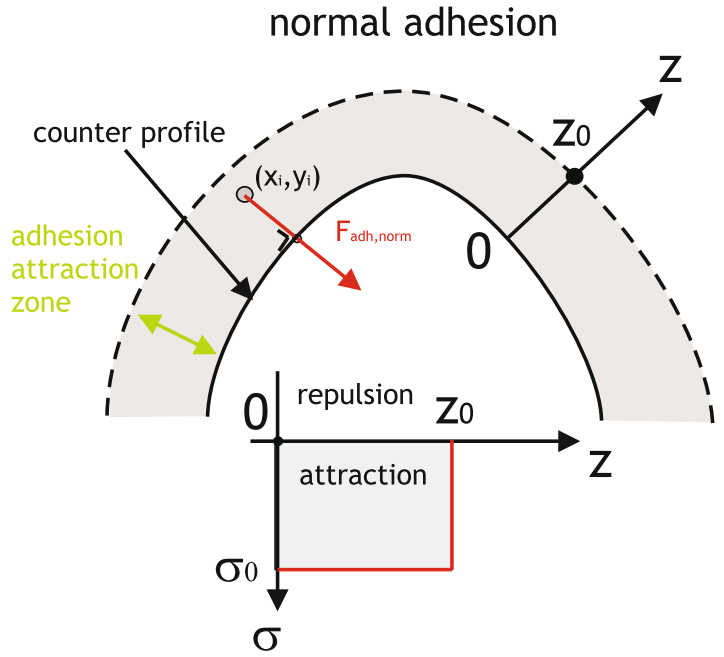




\subsection{Elastic-Plastic Asperity Behaviour}

Since the pioneering study of Greenwood and Williamson [13], numerous attempts to model the contact of rough surfaces were carried out. Several static models include the elastic-plastic behaviour of the contacting asperities [19, $34,24,23]$. On the other hand, viscoelastic materials can be modelled by combining basic mechanical models, such as the Voigt and Maxwell models. These elements consist of a spring and a dashpot in parallel and in series respectively to simulate the deformation behaviour of viscoelastic materials [35, 36]. In order to include the hysteretic elastic plastic spring behaviour in our model, a Maxwell-Slip element [37, 38] is used, which allows fast and accurate simulation of hysteretic behaviour of a weakening spring. The basic scheme of the Maxwell-Slip model is depicted in Fig. 3. The model consists of a parallel connection of elements, characterised by their stiffness $k_{j}$ and the maximum deformation of the spring $\Delta_{j}$ (with $j=1 \ldots M$ ). This latter parameter relates the statics of the element to its own frictional behaviour, as the maximal force, which the element can resist, is given by $F_{\max }=\Delta_{j} \cdot k_{j}$. As state variable, the spring deformation $\delta_{j}$ is chosen. When the element sticks, its position is fixed and the spring deformation $\delta_{j}$ increases, until its maximum value $\Delta_{j}$ is reached, as a result of which the element slips. Following Rizos and Fassois [39], the state equation for each element can be written in a dense way for both regimes as in Eq. 1:

$$
\begin{aligned}
\delta_{j}(t)= & \operatorname{sgn}\left\{x(t)-x(t-1)+\delta_{j}(t-1)\right\} \\
& \cdot \min \left\{\left|x(t)-x(t-1)+\delta_{j}(t-1)\right|, \Delta_{j}\right\},
\end{aligned}
$$

while the output is the sum of all spring forces:

$F(t)=\sum_{j=1}^{N} k_{j} \cdot \delta_{j}(t)$

This model structure is used to simulate the elastic-plastic behaviour of both the normal and tangential asperity deformation, one Maxwell-Slip model for each degree of freedom. The deformation of the spring is imposed by the motion of the asperity mass on the one hand, and the main mass position on the other hand. Every Maxwell-Slip model consists of two elements, of which one element is purely elastic (which can be considered as a Maxwell-Slip element with infinite maximum deformation), while the second is able to yield and thus to dissipate energy. This is depicted in Fig. 4, where the normal stiffness of an asperity is represented as the parallel connection of a purely elastic spring $k_{\mathrm{el}}$ and a Maxwell-Slip element with stiffness $k_{\mathrm{pl}}$. When applying a periodic deformation to the asperity in the normal direction, a force-deformation plot reveals the hysteresis behaviour, where the area enclosed by the hysteresis loop is a measure for the dissipated energy during the motion. Consequently, the asperity behaviour is dependent on the loading trajectory followed. Although the hysteresis model only comprises two elements, when considering a large amount of asperities with stochastically distributed parameters, no significant difference is observed when using more elements to model an asperity. Let us note, however, that during simulation, the motion of the asperity is not necessarily periodic, but depends on several model parameters, such as the asperity mass and stiffness, the motion of the main mass, and the shape of the counter profile. However, the mechanism of energy dissipation and hysteretic behaviour remains unaltered.

\section{Numerical Strategy and Calculation Method}

\subsection{Contact Detection}

The shape of the counter profile has to fulfil some conditions to yield a computationally efficient algorithm, without mortgaging the numerical stability more than necessary. The shape of the profile is of fixed pattern, with randomly varying wavelength, and constant amplitude. The
Fig. 3 The model structure of the Maxwell-Slip model

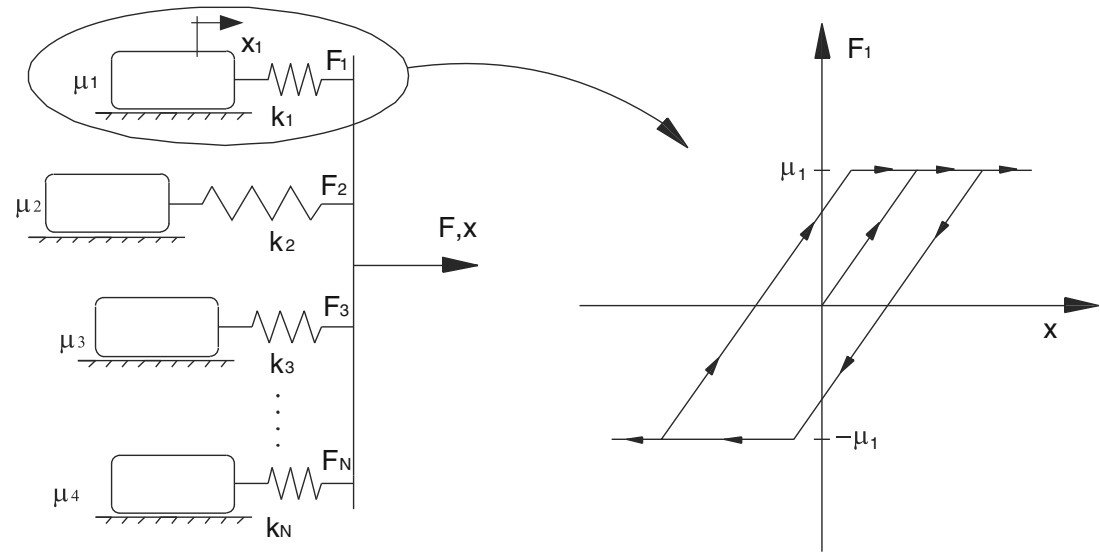




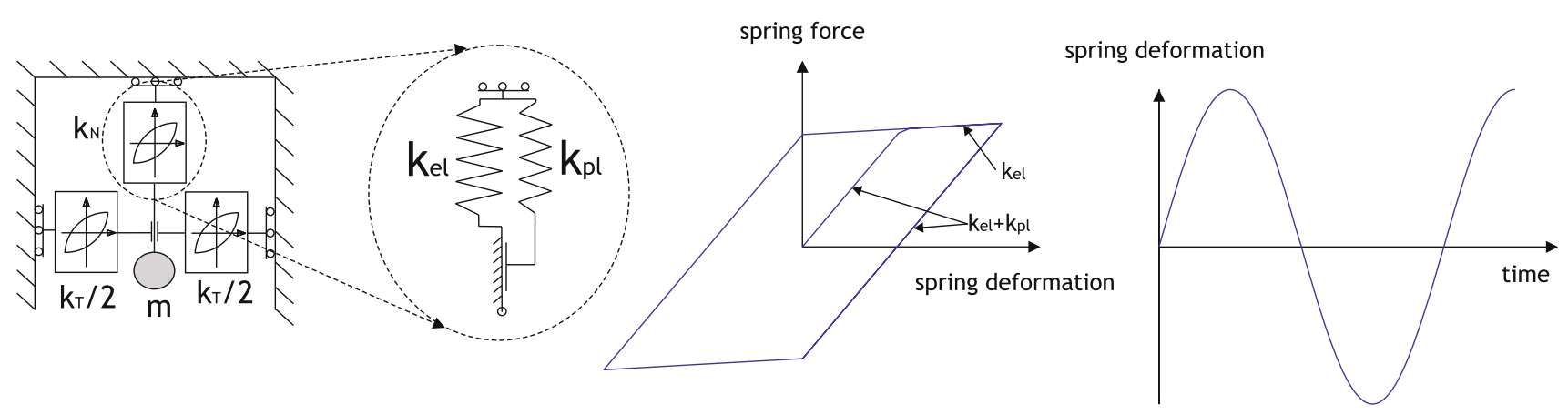

Fig. 4 The representation of an elastic-plastic asperity

choice for a counter profile with constant amplitude is beneficial, since the search algorithm - to find on which element of the counter profile penetration is possible-is abolished whenever the asperity is situated above the counter profile amplitude. Different possible shapes are depicted in Fig. 5. For the calculation of the reaction force of the profile on the asperity, the following procedure is used. When the asperity point mass interferes with the counter profile, the normal distance $\delta$ between the asperity mass position $\left(x_{i}, y_{i}\right)$ and the closest point $(x, y)$ on the counter profile is calculated. This is depicted in Fig. 6 for an arbitrary profile. The reaction force acts outwards along the line formed by $\left(x_{i}, y_{i}\right)$ and $(x, y)$. Obviously, the triangular (a) and trapezoidal profile (b) suffer from discontinuities at the edges, while for these same profiles, the interference depth is most readily computable as a pointline distance. The circular profile (d) also suffers from

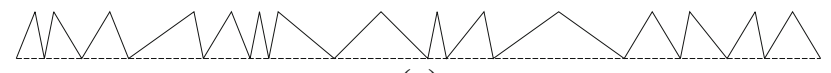

(a)

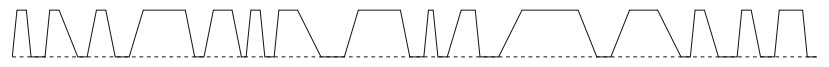

(b)

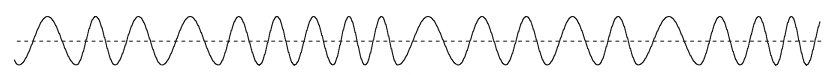

(c)

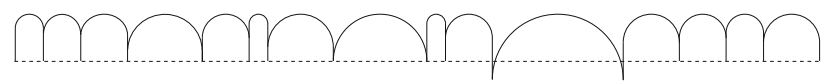

(d)

Fig. 5 Appropriate counter profiles. a Triangular, b trapezoidal, c sinusoidal and $\mathbf{d}$ circular

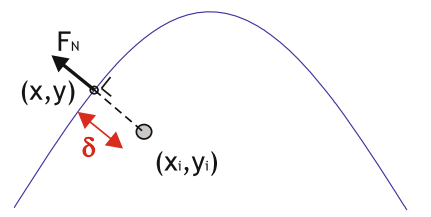

Fig. 6 Calculation of the interference depth $\delta$. The restoring force acts outwards via the normal vector on the profile
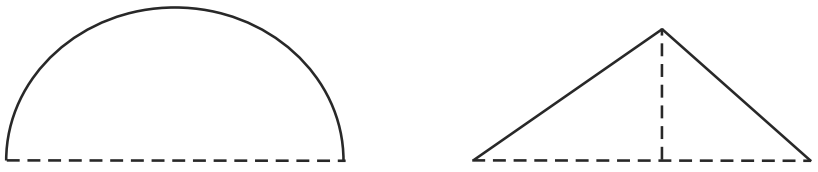

Fig. 7 Comparison of the circular and the triangular profile. The discontinuities are indicated by the dashed line. The circular profile has less discontinuities than the triangular

certain discontinuities, e.g. at the lower edge of the half circles, but these boundaries are less stringent. This is shown in Fig. 7. For the circular profile, the determination of the interference depth is computationally cheap as point-circle distance calculation. The sinusoidal profile (c) is optimal with regard to discontinuities, but computationally expensive, since the determination of the distance between a point and a sine curve requires a numerical root finding strategy, such as Newton-Raphson or bisection method.

\subsection{Model Equations}

Before setting out on the mathematical representation of the system, we refer to Fig. 1, where a global overview is given of the parameters included in the model. The equations of motion of this MDOF $m-c-k$ system are as follows (we refer to the nomenclature for an overview of the used symbols). For the main mass $M$, we have

$$
\begin{aligned}
M \ddot{x}_{t}= & K_{\mathrm{T}}\left(x_{m}-x_{t}\right)+C_{\mathrm{T}}\left(\dot{x}_{m}-\dot{x}_{t}\right)+\sum_{i=1}^{N} k_{\mathrm{T}, i}\left(x_{i}-x_{t}-d x_{\mathrm{T}, i}\right) \\
& +\ldots \sum_{i=1}^{N} c_{\mathrm{T}, i}\left(\dot{x}_{i}-\dot{x}_{t}\right) \\
M \ddot{y}_{t}= & \sum_{i=1}^{N} k_{\mathrm{N}, i}\left(y_{i}-y_{t}+l_{i}\right)+\sum_{i=1}^{N} c_{\mathrm{N}, i}\left(\dot{y}_{i}-\dot{y}_{t}\right)-F
\end{aligned}
$$

where $F$ is the external normal force applied onto the main mass. Depending on the excitation, the terms involving $K_{\mathrm{T}}$ and $C_{\mathrm{T}}$ in Eq. 3 are applicable or not. 
For the asperity masses, we have

$$
\begin{aligned}
m_{i} \ddot{x}_{i}= & k_{\mathrm{T}, i}\left(x_{t}-x_{i}+d x_{\mathrm{T}, i}\right)+c_{\mathrm{T}, i}\left(\dot{x}_{t}-\dot{x}_{i}\right) \\
& +\gamma k_{\mathrm{p}, i}\left(x-x_{i}\right)+F_{\mathrm{adh}, i, x} \\
m_{i} \ddot{y}_{i}= & k_{\mathrm{N}, i}\left(y_{t}-y_{i}-l_{i}\right)+c_{\mathrm{N}, i}\left(\dot{y}_{t}-\dot{y}_{i}\right) \\
& +\gamma k_{\mathrm{p}, i}\left(y-y_{i}\right)+F_{\mathrm{adh}, i, y}
\end{aligned}
$$

where $\gamma$ equals 1 if the asperity is in contact, and 0 otherwise. $F_{\mathrm{adh}, i, x}$ and $F_{\mathrm{adh}, i, y}$ are the $x$ and $y$ component, respectively, of the adhesion force affecting the $i$-th asperity, based on the normal or tangential adhesion law stated in Sect. 2.1.

In matrix representation, we can distinguish the following matrices:

$$
\begin{aligned}
& \mathbf{M}=\left[\begin{array}{cccccc}
M & & & & & 0 \\
& m_{1} & \ddots & & \ldots & \\
& \ddots & \ddots & 0 & & \\
& & 0 & M & \ddots & \\
& \ldots & & \ddots & m_{1} & \\
0 & & & & & \ddots
\end{array}\right] \\
& \mathbf{K}=\left[\begin{array}{cccccc}
K+\sum k_{\mathrm{T}, i} & -k_{\mathrm{T}, 1} & \cdots & 0 & \cdots & 0 \\
-k_{\mathrm{T}, 1} & k_{\mathrm{T}, 1}+\gamma k_{\mathrm{p}, 1} & 0 & \vdots & \ddots & \vdots \\
\vdots & 0 & \ddots & 0 & \cdots & 0 \\
0 & \cdots & 0 & \sum k_{\mathrm{N}, i} & -k_{\mathrm{N}, 1} & \vdots \\
\vdots & \ddots & \vdots & -k_{\mathrm{N}, 1} & k_{\mathrm{N}, 1}+\gamma k_{\mathrm{p}, 1} & 0 \\
0 & \cdots & 0 & \ldots & 0 & \ddots
\end{array}\right]
\end{aligned}
$$

$$
\mathbf{C}=\left[\begin{array}{cccccc}
C+\sum c_{\mathrm{T}, i} & -c_{\mathrm{T}, 1} & \cdots & 0 & \cdots & 0 \\
-c_{\mathrm{T}, 1} & c_{\mathrm{T}, 1} & 0 & \vdots & \ddots & \vdots \\
\vdots & 0 & \ddots & 0 & \cdots & 0 \\
0 & \cdots & 0 & \sum c_{\mathrm{N}, i} & -c_{\mathrm{N}, 1} & \vdots \\
\vdots & \ddots & \vdots & -c_{\mathrm{N}, 1} & c_{\mathrm{N}, 1} & 0 \\
0 & \cdots & 0 & \cdots & 0 & \ddots
\end{array}\right]
$$

$$
\mathbf{F}=\left[\begin{array}{c}
K x_{m}+C \dot{x}_{m}-\sum k_{\mathrm{T}, i} d x_{\mathrm{T}, i} \\
F_{\mathrm{adh}, 1, x}+k_{\mathrm{T}, 1} d x_{\mathrm{T}, 1}+\gamma k_{\mathrm{p}, 1} x_{\mathrm{p}, 1} \\
\vdots \\
\sum k_{\mathrm{N}, i} l_{i}-F \\
F_{\mathrm{adh}, 1, y}-k_{\mathrm{N}, 1} l_{1}+\gamma k_{\mathrm{p}, 1} y_{\mathrm{p}, 1} \\
\vdots
\end{array}\right]
$$

This results in

$$
\dot{\mathbf{q}}=\left[\begin{array}{cc}
\mathbf{0} & \mathbf{I} \\
\mathbf{M}^{-1} \mathbf{K} & \mathbf{M}^{-1} \mathbf{C}
\end{array}\right] \cdot \mathbf{q}+\mathbf{M}^{-1}\left[\begin{array}{c}
\mathbf{0} \\
\mathbf{F}
\end{array}\right]
$$

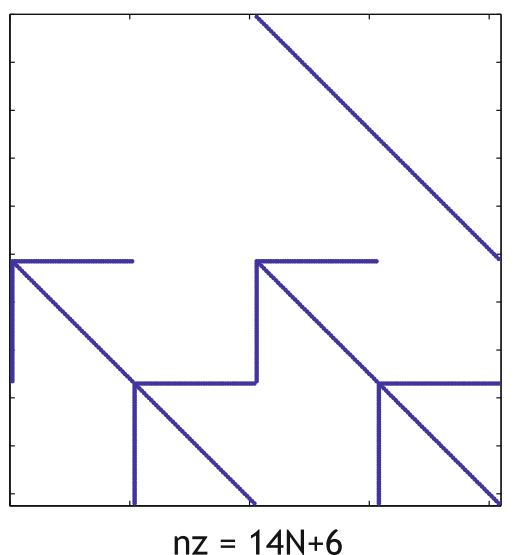

Fig. 8 Sparsity pattern of the matrix $\mathbf{A}$. The size of the matrix is $(4 N+4)$ by $(4 N+4)$, with $N$ the number of asperities in the model

$\dot{\mathbf{q}}=\mathbf{A q}+\mathbf{B}$

with

$$
\begin{aligned}
\mathbf{q} & =\left[x_{t}, x_{1}, \ldots, y_{t}, y_{1}, \ldots, \dot{x}_{t}, \dot{x}_{1}, \ldots, \dot{y}_{t}, \dot{y}_{1}, \ldots\right]^{T} \\
\dot{\mathbf{q}} & =\left[\dot{x}_{t}, \dot{x}_{1}, \ldots, \dot{y}_{t}, \dot{y}_{1}, \ldots, \ddot{x}_{t}, \ddot{x}_{1}, \ldots, \ddot{y}_{t}, \ddot{y}_{1}, \ldots\right]^{T}
\end{aligned}
$$

In Fig. 8, the sparsity pattern of the matrix $\mathbf{A}$ is depicted, which is beneficial when applying dedicated sparse-matrix techniques during the calculations. In general, at least 2000 asperities are used in the simulations to obtain reasonable frictional behaviour.

\subsection{A Dimensionless Approach}

We consider it useful first to normalise the problem with respect to its parameters. The problem contains a large number of parameters, thus a reduction of the number of system variables permits an easier analysis of the results, and gives better insight into the behaviour. This parameter reduction is attained by the application of the VaschyBuckingham- $\Pi$ theorem [40].

We define the reference angular frequency $\omega_{N}$ to normalise the time:

$$
\begin{aligned}
\tau & =\omega_{\mathrm{N}} t \quad \omega_{\mathrm{N}}=\sqrt{\left(\sum k_{\mathrm{T}}+\Sigma k_{\mathrm{N}}\right) / M} \\
\mathrm{~d} \tau & =\omega_{\mathrm{N}} \mathrm{d} t \\
\frac{\mathrm{d} \tau}{\mathrm{d} t} & =\omega_{\mathrm{N}} \\
\ddot{x} & \rightarrow x^{\prime \prime}
\end{aligned}
$$

with accents denoting derivatives with respect to $\tau$.

Further, we normalise the displacements by the mean counter profile wavelength $\lambda$ :

or 


$$
\begin{aligned}
X & =\frac{x}{\lambda} \\
Y & =\frac{y}{\lambda} \\
L_{i} & =\frac{l_{i}}{\lambda}
\end{aligned}
$$

Substituting Eqs. 12 and 13 in Eqs. 5 and 4 and rearranging, we obtain

$$
\begin{aligned}
X_{t}^{\prime \prime}= & \frac{K_{\mathrm{T}}\left(X_{m}-X_{t}\right)}{M \omega_{\mathrm{N}}^{2}}+\frac{C_{\mathrm{T}} \omega_{\mathrm{N}}\left(X_{m}^{\prime}-X_{t}^{\prime}\right)}{M \omega_{\mathrm{N}}^{2}} \\
& +\frac{\sum_{i=1}^{N} k_{\mathrm{T}, i}\left(X_{i}-X_{t}-\mathrm{d} X_{\mathrm{T}, i)}\right.}{M \omega_{\mathrm{N}}^{2}}+\frac{\sum_{i=1}^{N} \omega_{\mathrm{N}} c_{\mathrm{T}, i}\left(X_{i}^{\prime}-X_{t}^{\prime}\right)}{M \omega_{\mathrm{N}}^{2}} \\
Y_{t}^{\prime \prime}= & \frac{\sum_{i=1}^{N} k_{\mathrm{N}, i}\left(Y_{i}-Y_{t}+L_{i}\right)}{M \omega_{\mathrm{N}}^{2}}+\frac{\sum_{i=1}^{N} \omega_{\mathrm{N}} c_{\mathrm{N}, i}\left(Y_{i}^{\prime}-Y_{t}^{\prime}\right)}{M \omega_{\mathrm{N}}^{2}} \\
& -\frac{F}{M \omega_{\mathrm{N}}^{2} \lambda}
\end{aligned}
$$

and

$$
\begin{aligned}
X_{i}^{\prime \prime}= & \frac{k_{\mathrm{T}, i}\left(X_{t}-X_{i}+\mathrm{d} X_{\mathrm{T}, i}\right)}{m_{i} \omega_{\mathrm{N}}^{2}}+\frac{\omega_{\mathrm{N}} c_{\mathrm{T}, i}\left(X_{t}^{\prime}-X_{i}^{\prime}\right)}{m_{i} \omega_{\mathrm{N}}^{2}} \\
& +\frac{\gamma k_{\mathrm{p}, i}\left(X-X_{i}\right)}{m_{i} \omega_{\mathrm{N}}^{2}}+\frac{F_{\mathrm{adh}, i, x}}{m_{i} \omega_{\mathrm{N}}^{2} \lambda} \\
Y_{i}^{\prime \prime}= & \frac{k_{\mathrm{N}, i}\left(Y_{t}-Y_{i}-L_{i}\right)}{m_{i} \omega_{\mathrm{N}}^{2}}+\frac{\omega_{\mathrm{N}} c_{\mathrm{N}, i}\left(Y_{t}^{\prime}-Y_{i}^{\prime}\right)}{m_{i} \omega_{\mathrm{N}}^{2}} \\
& +\frac{\gamma k_{\mathrm{p}, i}\left(Y-Y_{i}\right)}{m_{i} \omega_{\mathrm{N}}^{2}}+\frac{F_{\mathrm{adh}, i, y}}{m_{i} \omega_{\mathrm{N}}^{2} \lambda}
\end{aligned}
$$

With

$$
\begin{aligned}
K_{\mathrm{T}} & =\omega_{\mathrm{nT}}^{2} M \\
C_{\mathrm{T}} & =2 \zeta M \omega_{\mathrm{nT}} \\
k_{\mathrm{T}, i} & =\omega_{\mathrm{nT} i}^{2} m_{i} \\
k_{\mathrm{N}, i} & =\omega_{\mathrm{nN} i}^{2} m_{i} \\
k_{\mathrm{p}, i} & =\omega_{\mathrm{nP} i}^{2} m_{i} \\
c_{\mathrm{T}, i} & =2 \zeta_{\mathrm{T}, i} m_{i} \omega_{\mathrm{nT} i} \\
c_{\mathrm{N}, i} & =2 \zeta_{\mathrm{N}, i} m_{i} \omega_{\mathrm{nN} i}
\end{aligned}
$$

Simplifying further, we obtain the final form:

$$
\begin{aligned}
X_{t}^{\prime \prime}= & \frac{\omega_{\mathrm{nT}}^{2}}{\omega_{\mathrm{N}}^{2}}\left(X_{m}-X_{t}\right)+2 \zeta \frac{\omega_{\mathrm{nT}}}{\omega_{\mathrm{N}}}\left(X_{m}^{\prime}-X_{t}^{\prime}\right) \\
& +\ldots \frac{\sum_{i=1}^{N} m_{i} \omega_{\mathrm{nT} i}^{2}\left(X_{i}-X_{t}-\mathrm{d} X_{\mathrm{T}, i}\right)}{M \omega_{\mathrm{N}}^{2}} \\
+ & \frac{\sum_{i=1}^{N} 2 \zeta_{\mathrm{T}, i} m_{i} \omega_{\mathrm{nT} i}\left(X_{i}^{\prime}-X_{t}^{\prime}\right)}{M \omega_{\mathrm{N}}} \\
Y_{t}^{\prime \prime}= & \frac{\sum_{i=1}^{N} m_{i} \omega_{\mathrm{nN} i}^{2}\left(Y_{i}-Y_{t}+L_{i}\right)}{M \omega_{\mathrm{N}}^{2}} \\
+ & \frac{\sum_{i=1}^{N} 2 \zeta_{\mathrm{N}, i} m_{i} \omega_{\mathrm{nN} i}\left(Y_{i}^{\prime}-Y_{t}^{\prime}\right)}{M \omega_{\mathrm{N}}}-\frac{F}{M \omega_{\mathrm{N}}^{2} \lambda}
\end{aligned}
$$

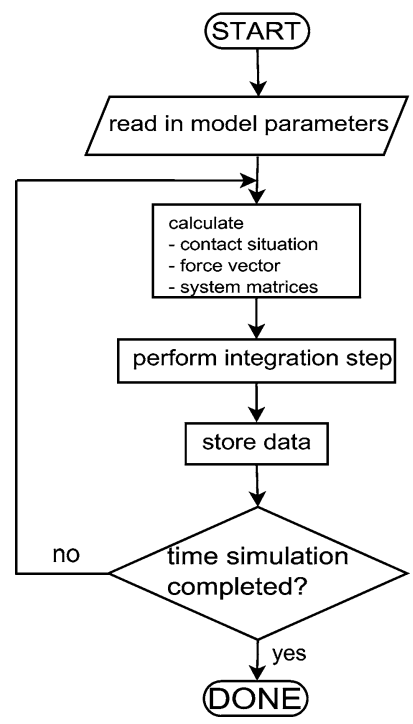

Fig. 9 Flowchart of the solution strategy

and

$$
\begin{aligned}
X_{i}^{\prime \prime}= & \frac{\omega_{\mathrm{nT} i}^{2}}{\omega_{\mathrm{N}}^{2}}\left(X_{t}-X_{i}+\mathrm{d} X_{\mathrm{T}, i}\right)+2 \zeta_{\mathrm{T}, i} \frac{\omega_{\mathrm{nT} i}}{\omega_{\mathrm{N}}}\left(X_{t}^{\prime}-X_{i}^{\prime}\right) \\
& +\gamma \frac{\omega_{\mathrm{nP} i}^{2}}{\omega_{\mathrm{N}}^{2}}\left(X-X_{i}\right)+\frac{F_{\mathrm{adh}, i, x}}{m_{i} \omega_{\mathrm{N}}^{2} \lambda} \\
Y_{i}^{\prime \prime}= & \frac{\omega_{\mathrm{nN} i}^{2}}{\omega_{\mathrm{N}}^{2}}\left(Y_{t}-Y_{i}-L_{i}\right)+2 \zeta_{\mathrm{N}, i} \frac{\omega_{\mathrm{nN} i}}{\omega_{\mathrm{N}}}\left(Y_{t}^{\prime}-Y_{i}^{\prime}\right) \\
& +\gamma \frac{\omega_{\mathrm{nP} i}^{2}}{\omega_{\mathrm{N}}^{2}}\left(Y-Y_{i}\right)+\frac{F_{\mathrm{adh}, i, y}}{m_{i} \omega_{\mathrm{N}}^{2} \lambda}
\end{aligned}
$$

The nonlinear constraints of the problem, such as the adhesion, the contact formation and loss, and the hysteretic spring behaviour of the asperities, hamper the solving of the system of ODEs. Therefore, an Adams-Bashford-Adams-Moulton predictor-corrector method is used. (Other numerical solvers may also be appropriate to deal with the nonlinear behaviour of the system [41]). Structural dynamics solvers, such as Newmark alpha-beta, suffer from the fact that matrix inversion is necessary for these implicit methods. Since the stiffness matrix changes owing to the elastic-plastic asperity behaviour, this inversion needs to be carried out at almost every time step, which is computationally intensive for large, though sparse, systems. In Fig. 9, a flowchart shows the different steps of the solution strategy.

\section{Numerical Results}

This section presents the basic friction characteristics, as simulated by the model described above, and compares them to experimentally observed characteristics (where known). This comparison is per se qualitative since the model contains a large number of unknown parameters, 
which have to be determined when a quantitative comparison is sought (see Sect. 4.11, for a discussion of this point). The simulations also serve to check the performance and validity of the model. We refer to the Appendix for an overview of the numerical values of the used model parameters, unless otherwise stated in this section. These model parameters can generally be obtained from an identification procedure, based on experimental data, combined with foreknowledge of the nature of the surfaces in contact regarding material and geometrical properties.

Worthy of note is that the correspondence between physical and geometrical properties of the rubbing materials on the one hand, and the model parameters, on the other, is not a straightforward one (see Sect. 4.11). In order to transform physical and geometrical parameters into model parameters, other assumptions have to be made, which are outside the scope of this article. Our purpose here consists mainly in demonstrating how a model formulation such as ours can yield all the known frictional characteristics using arbitrary parameters.

\subsection{Friction Force in Function of the Sliding Velocity: the Stribeck Curve}

This is the most well-known friction characteristic, manifesting itself in both dry and lubricated contacts. With increasing sliding velocity, the friction coefficient, generally, first decreases (velocity weakening) and then increases (velocity strengthening, or viscous effect). Since adhesion forces play a major role in this phenomenon, we have exploited the simulation also to make a comparison between the two adhesion laws postulated in our model (namely, tangential versus normal adhesion law).

In Fig. 10, the results of a simulation of the static Stribeck curve is shown for the model with normal adhesion law and tangential adhesion law. Although the normal adhesion law can be considered as a more classical and correct approach, both the normal and tangential adhesion laws yield qualitatively similar results. Therefore, one can conclude that the behaviour using either approaches is qualitatively of the same kind, which is also confirmed by other simulations. The presented results hereafter are based on the model with the tangential adhesion law, which is numerically less demanding.

\subsection{Normal Creep}

When putting two surface in contact, the actual contact regions are extremely small compared to the apparent contact area. It is, therefore, reasonable to assume that certain asperities in contact will deform plastically. Consequently, the contacting bodies will creep under external load $[42,43]$. The hardness tests of Mulhearn and Tabor [44] show similar behaviour. Bowden and Tabor [45] describe the phenomenon of indentation hardness decrease as similar to the viscous creep process in tensile tests. Gitis and Volpe [46] study the static friction mechanism at short and long term dwell. The stiction models they reviewed, cover processes such as material deformation, meniscus formation and viscous lubricant flow. They also give an overview of the various formulas for the time-dependency of the static friction force. Berthoud and Baumberger [47] investigate the time-strengthening and velocity weakening friction of polymer glass and study the role of asperity creep under normal load. Persson $[9,10]$ states that the increase in contact area with time of stationary contact is responsible for an increasing static friction force with contact time.

The presented generic model can be tested for this phenomenon by the following simulation experiment. The upper mass (having the asperities) is brought into stationary contact with the counter profile. A certain constant normal force is applied externally to the main mass. The normal position $Y_{t}$ of the main mass is plotted as function of the contact time in Fig. 11. One can observe the decreasing vertical height of the main mass as function of
Fig. 10 A comparison of the Stribeck curve simulated with the normal adhesion law (left panel) and the tangential adhesion law (right panel). The ordinate is the normalised friction force, while the abscissa is the normalised sliding velocity. Weakening and strengthening are clearly in evidence

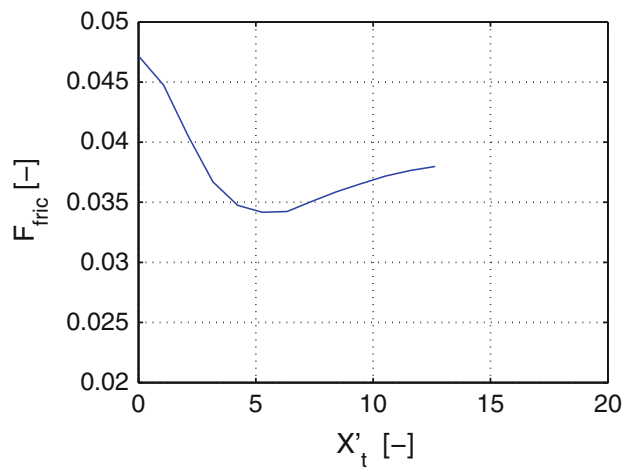

(a)

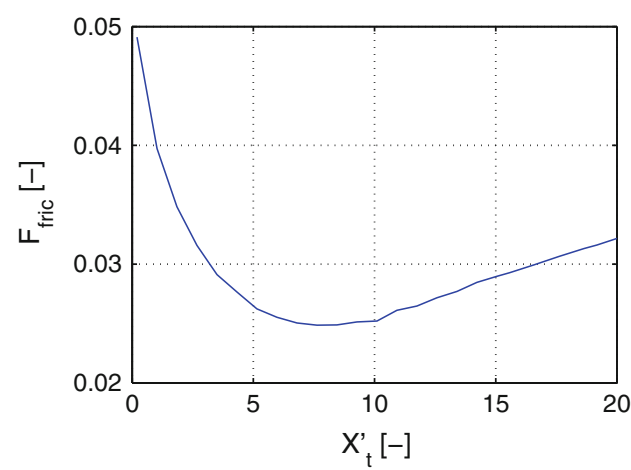

(b) 


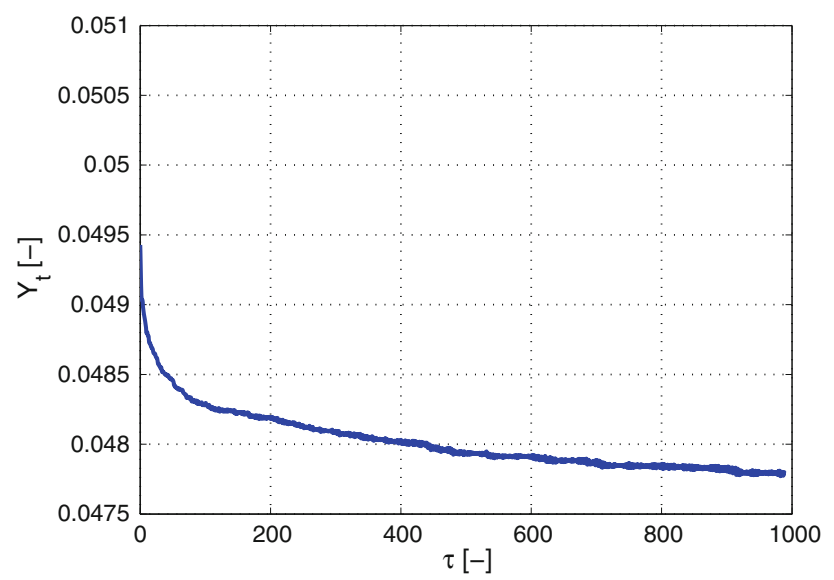

Fig. 11 Normal creep simulation

the contact time; initially very fast (high slope), but with decreasing slope as time elapses. This compares favourably with experimental results from literature [48].

\subsection{Static Coefficient of Friction as Function of Stick Time}

The preceding study of the normal creep with contact time points out the fact that the loaded asperities never reach a strict equilibrium in finite time. This time-dependency reveals itself in an increasing static coefficient of friction as function of stick or dwell time. Coulomb [4, 49] was probably the first to discover this dependency. Gitis and Volpe [46] list some important equations from literature.

The static friction coefficient $\mu_{\mathrm{s}}$ is found experimentally, to increase in a quasi-logarithmic way with the stick time $\tau$, for a wide range of material combinations, according to Eq. 19 [43, 48, 50, 51]. This can be explained by the relationship between the strengthening with aging of the contact junctions, and the plastic relaxation of the loaded contacts, which results in an increase of the real contact area and consequently to an increase of the static coefficient of friction [52].

$\mu_{\mathrm{s}}(\tau)=\mu_{0}+\beta \ln \tau$

For the simulations with the presented model, the system is preloaded with a force and subsequently kept stationary for a certain sticktime $\tau$. Afterwards, a constant velocity is applied and the maximum pre-sliding friction force is captured and used to calculated the static coefficient of friction $\mu_{\mathrm{s}}$. The results are plotted in Fig. 12.

\subsection{Contact Simulation Indentation Simulation}

The normal indentation simulation is carried out by applying a (position controlled) forced indentation to the system while investigating the normal contact force. In

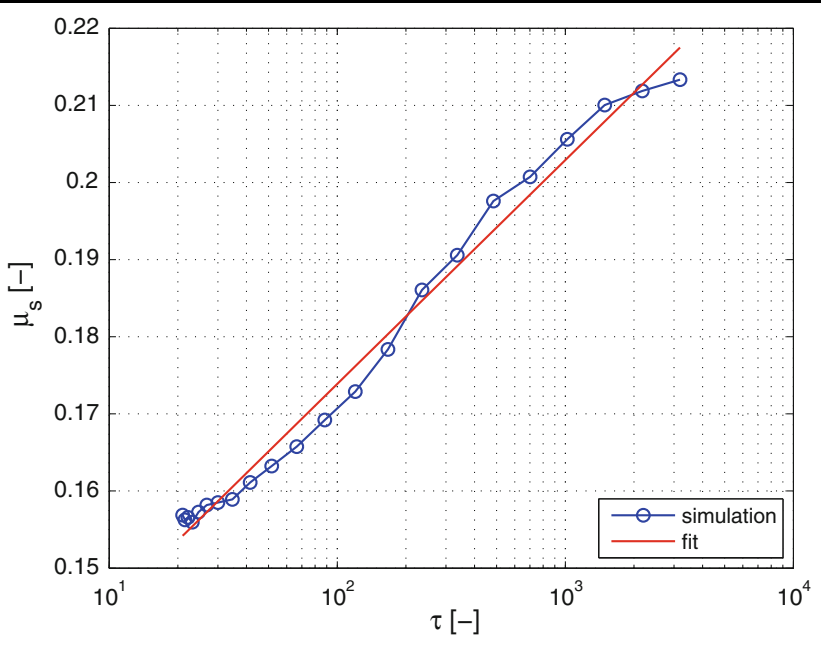

Fig. 12 Static friction coefficient $\mu_{\mathrm{s}}$ as function of the stick time $\tau$ prior to sliding. The simulation results are compared to the fit with the model Eq. 19

Fig. 13a and b, the normal displacement $Y_{t}$ and the normal force $F_{\text {norm }}$ are depicted as function of time, while Fig. 13c presents the evolution of the normal force as function of the normal indentation. The arrows in Fig. 13c indicate how the loop is passed through. The slope of the curve increases first with raising indentation, due to the increasing number of asperities in contact. Subsequently, plastic yielding causes a diminution of that increase. At the reversal point of indentation, a steep return is noticed in the forceindentation curve. One can also notice the oscillatory behaviour of the normal force at the end of the simulation $(t>12[-])$, due to the low damping in the system. The linear damping terms $\zeta_{\mathrm{N}, i}$ and $\zeta_{\mathrm{T}, i}$ are set to zero in these experiments, and thus the only damping in the system originates from the elastic-plastic spring behaviour, which reduces with decreasing interference depth $Y_{t}$ (Fig. 12).

\subsection{Pre-Sliding Hysteresis}

Prior to gross sliding, the tangential contact force is a (hysteresis) function of the tangential displacement. In Fig. 14, the pre-sliding hysteresis $F_{\text {fric }}=\mathcal{F}\left(X_{t}\right)$ is depicted for different excitation frequencies $f_{t}$. One can notice the somewhat little dependence of the hysteresis curve on the frequency of excitation, which was also observed experimentally by Lampaert et al. [53]. The evolution of the hysteresis curve with increasing excitation amplitude $X_{t}$, is depicted in Fig. 15, where the transition from pre-sliding to sliding hysteresis is visualised. In Fig. 16, experimental evidence is shown of a hysteresis measurement for different tangential strokes [62], presenting similar behaviour, however only in the pre-sliding region.

In Fig. 17, a hysteresis simulation is presented in which the wiping out effect [54] is demonstrated. When the 
Fig. 13 Normal loading. A vertical motion $Y_{t}$, depicted in $\mathbf{a}$, is imposed to the main mass. The resulting normal contact force is depicted in $\mathbf{b}$, while the force-displacements curve is presented in c. The area of the loop determines the unrecoverable dissipated energy

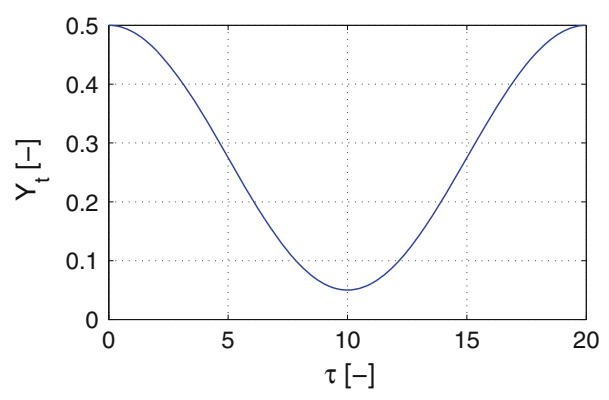

(a)

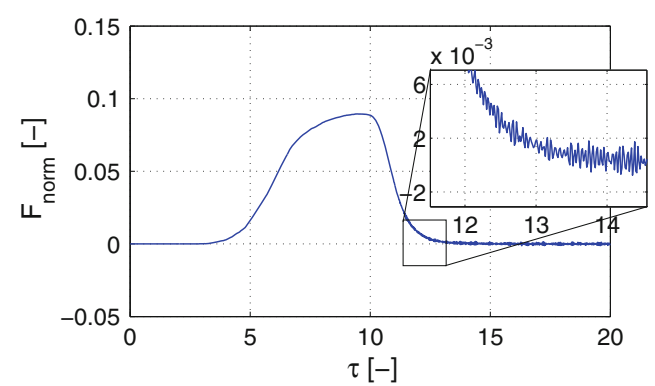

(b)

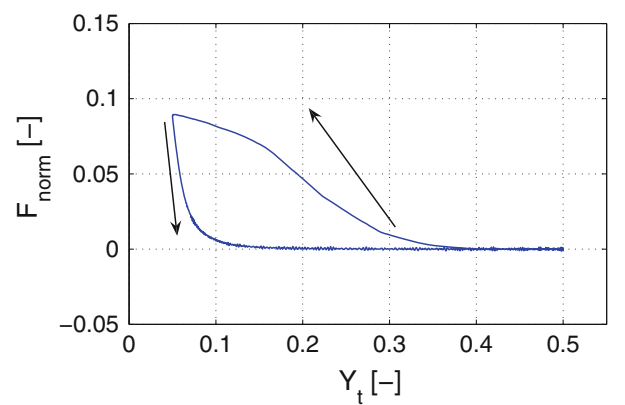

(c)

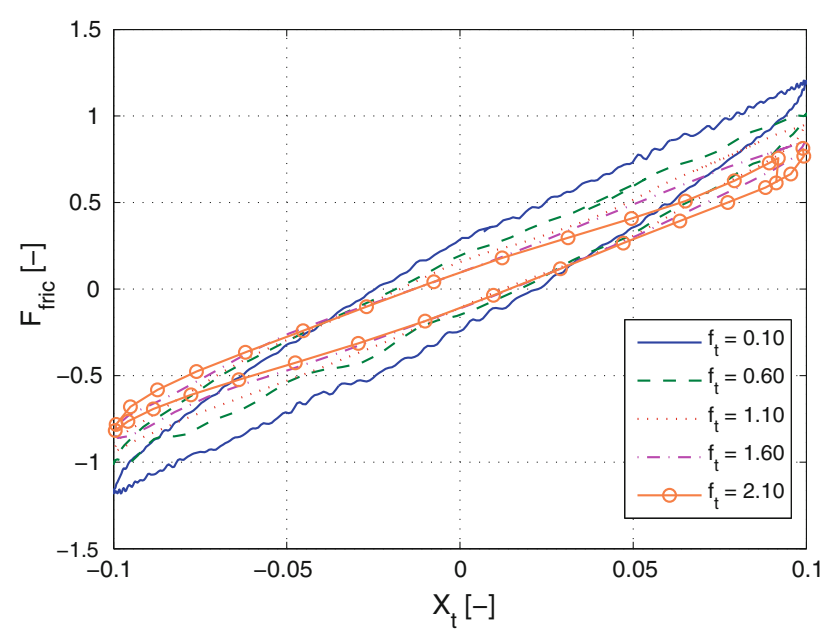

Fig. 14 The frequency independence of the pre-sliding hysteresis loop

motion is reversed before the hysteresis loop is closed, a new internal loop is formed. When reversing the motion sense once more, the internal loop is closed when arriving at the previous reversal point. After closing the internal loop, the path of the original hysteresis curve is followed and the effect of the two past reversal points has no longer effect [55]. The left panel 17 shows the continuous excitation signal $X_{\mathrm{t}}$ which is applied to the system, while the right panel 17 shows the external hysteresis loop with the internal loop. From this, one can conclude that this type of hysteresis is "hysteresis with nonlocal memory", following the "Masing Rules" [56, 57, 58, 55].

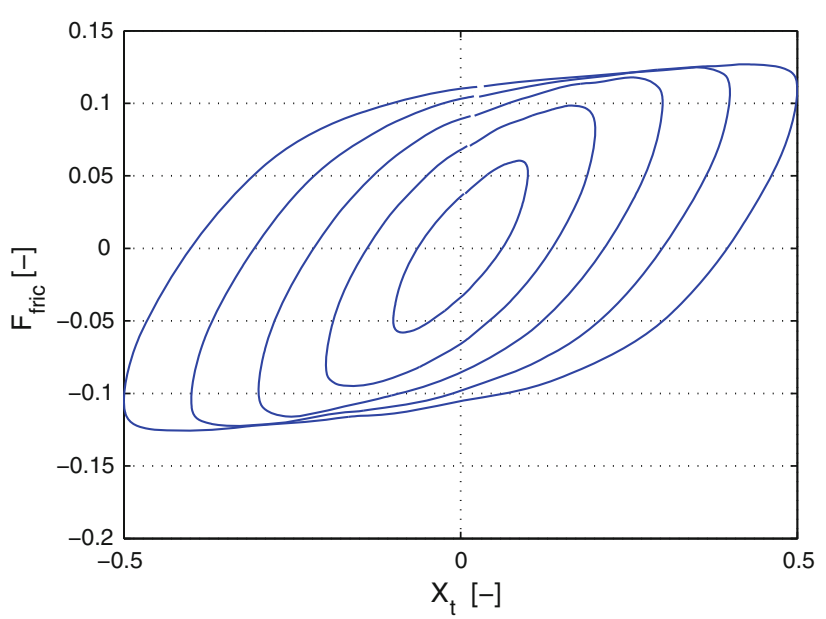

Fig. 15 The amplitude dependency of the hysteresis loop

\subsection{Gross Sliding and Transition Behaviour}

In this section, the model results are described for the transition from presiding to sliding regime and vice versa. In order to perform these experiments, a sinusoidal position excitation is applied to the main mass. In Fig. 18, this transition behaviour is depicted. The friction force is shown as function of the velocity. This behaviour is not only dependent on the chosen model parameters, but also on the excitation signal and its evolution with time (represented in our simulation by loops with increasing frequency). Consequently, a large diversity is observed in this type of behaviour. In order to confirm these results, experimental 


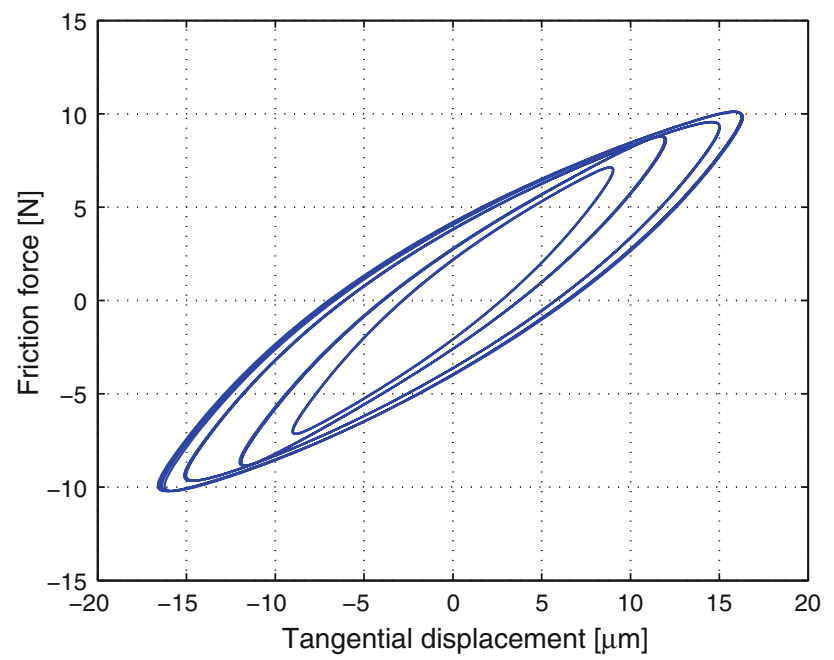

Fig. 16 Hysteresis measurement for different tangential strokes, PET plastic on aluminium, $1 \mathrm{~Hz}$ command signal, $68 \mathrm{~N}$ normal load

evidence is shown in Fig. 19 for the contact of steel on PET plastic. Similar results were obtained in the experimental studies by Kappagantu and Feeny [59] and Yanada and Sekikawa [60].

\subsection{Effect of the Normal Load on the Coefficient of Sliding Friction}

In this simulation, the effect of the normal load on the coefficient of friction $\mu$ is investigated. In Fig. 20a, the evolution of $\mu$ as function of normal load is depicted in the pre-sliding regime. One can notice the trend of decreasing coefficient of friction with increasing normal load. A similar tendency was obtained in another simulation study [61]. For higher loads, the static friction coefficient approaches a constant value. Experimental studies on a linear test setup [62] show results of the same kind. In Fig. 20b, similar simulation results are depicted up to sliding of the main mass, where also a decreasing trend of the coefficient of friction is noticed with increasing normal load.

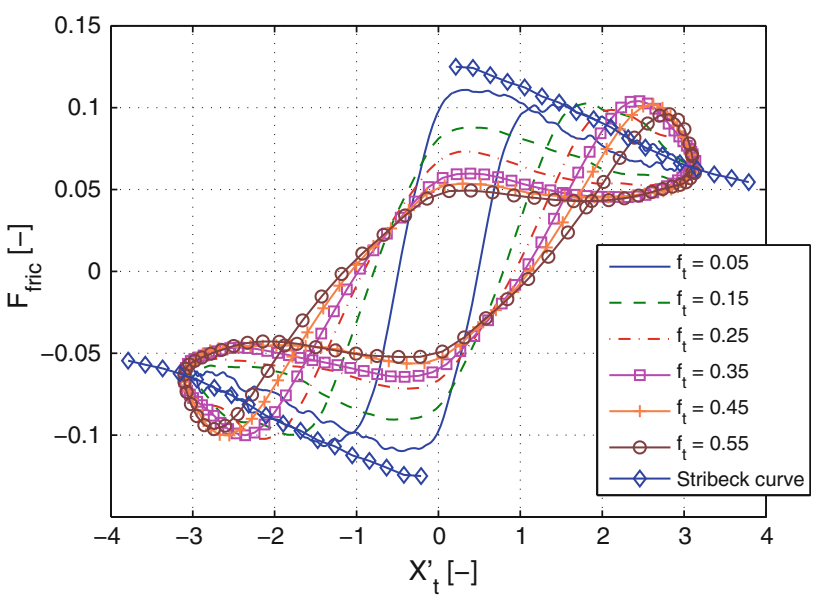

Fig. 18 Friction force as function of velocity for different excitation frequencies. In addition, the (static) Stribeck curve is plotted for reference

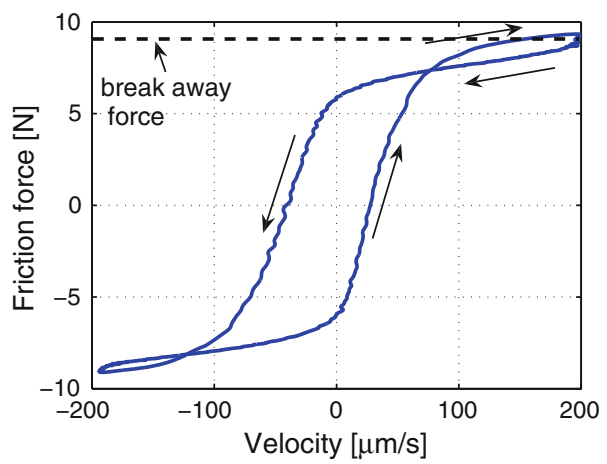

Fig. 19 Experimental results of friction lag for steel on PET plastic, $0.2 \mathrm{~Hz}$ command with a normal load $W$ of $48 \mathrm{~N}$

\subsection{Sliding Friction Force Dynamics}

In this section, the model results are presented regarding the static Stribeck curve and the dynamic friction lag simulations. Figure 21 shows the friction force as function of the velocity. The solid line represents the Stribeck curve,
Fig. 17 The nonlocal memory effect. In a, the excitation signal $X_{t}$ is depicted as function of time, while $\mathbf{b}$ shows the hysteresis loop with the internal loop

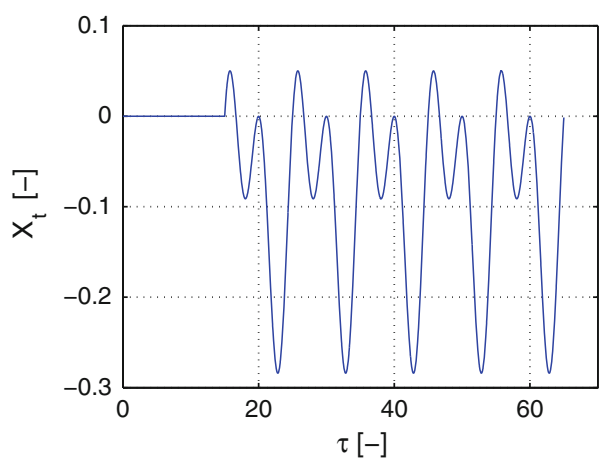

(a)

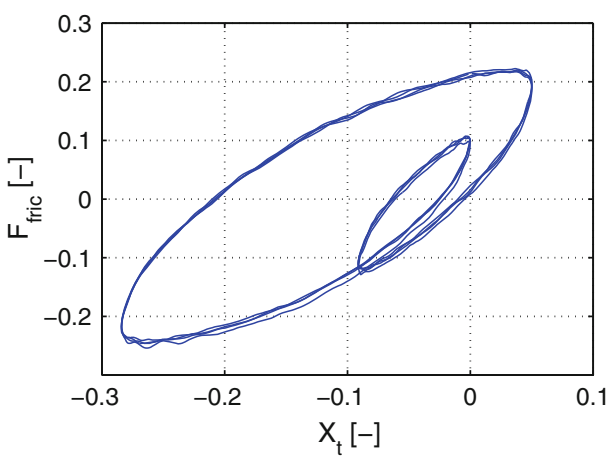

(b) 


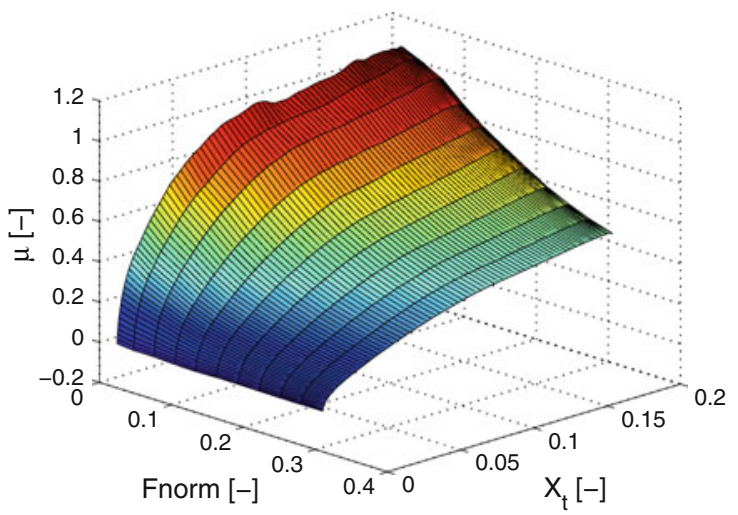

(a)

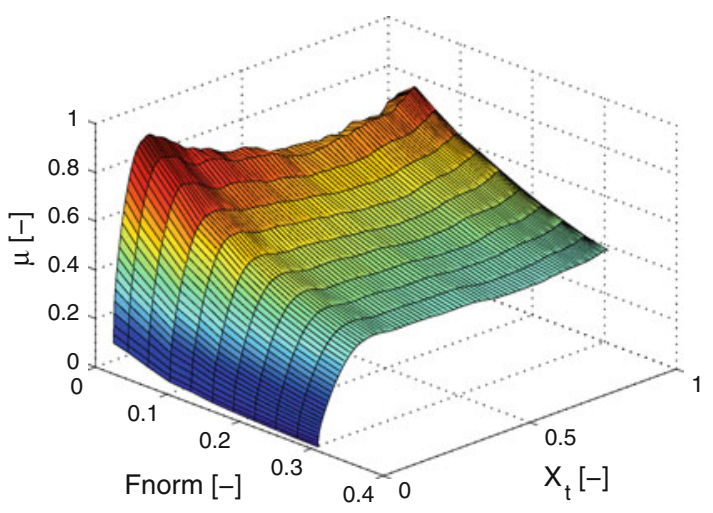

(b)

Fig. 20 Effect of the normal load on the pre-sliding coefficient of friction. a In pre-sliding, $\mathbf{b}$ up to gross sliding. The trend is a decreasing coefficient of friction at each section $X_{t}=$ constant

obtained by applying constant velocities to the main mass. The curve shows the combination of velocity weakening effect (decreasing friction force as function of increasing steady state velocity, Stribeck effect) and the velocity strengthening effect (increasing friction force as function of increasing steady state velocity, 'viscous' effect).

Frictional lag, also called frictional memory or hysteresis in the velocity, is also shown in Fig. 21. In order to obtain the friction-lag loops shown in Fig. 21, a sinusoidal excitation superposed on a constant offset velocity, is applied as position input to the main mass. From the results, one can see how the dynamic force exceeds the steady state curve when the mass accelerates, while the dynamic force is lower than the steady state curve

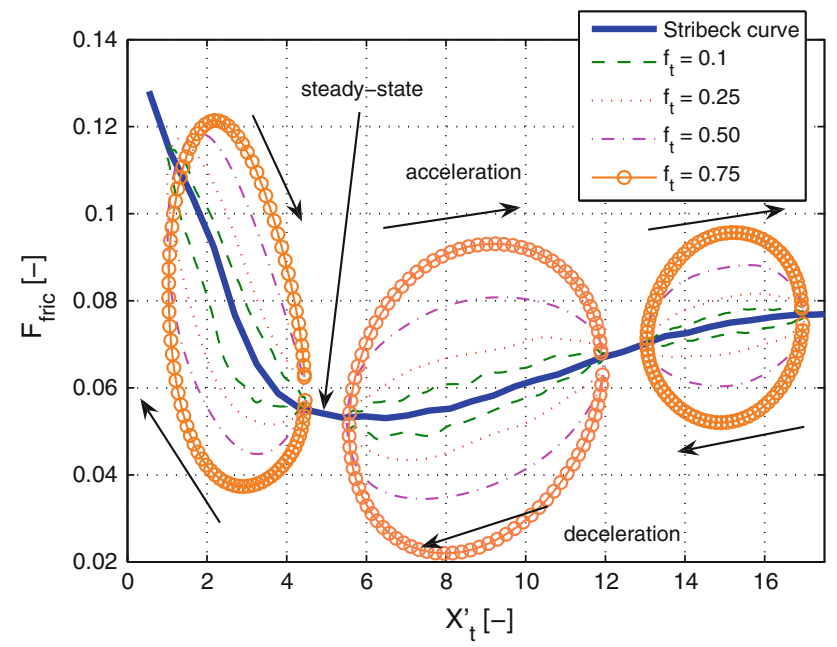

Fig. 21 The normalised friction force as function of the normalised velocity. The steady state curve as well as some non-steady state loops, for different excitation frequencies and around three different sliding velocities, are depicted during deceleration. One can also notice how the loop widens in the vertical direction (higher variation in friction force) when the frequency of excitation is increased. The behaviour is observed experimentally by Baumberger [48, 50] and is similar to that obtained in lubricated friction $[63,64]$.

\subsection{Dynamic Behaviour in the Normal Direction}

Recently, experimental evidence was shown that there is a regular normal displacement associated with the tangential motion, describing rate-independent hysteresis butterfly curves in dry friction, similar in nature to the piezoelectric and magnetic hysteresis [65]. These ideas date back to Amontons [2], who set up the hypothesis that the friction originates from the climbing of asperities one upon the other, and Coulomb [4], who explained the difference between the static and kinetic friction forces by his brush analogy. He proposed that, when the contacting surfaces are left to rest under a normal load, the contacting asperities will sink like brushes, increasing the engagement of the contacting surfaces. Tolstoi [66] explained the relative normal displacement by the oscillatory behaviour of the asperities, due to their nonlinear stiffness characteristics. In this model, both approaches are reconciled, as shown in the model description. In Fig. 22, three hysteresis plots are depicted, together with the imposed motion on the main mass. The well known hysteresis of tangential force as function of tangential displacement is shown in Fig. 22b. Figure 22c and $d$ shows the butterfly hysteresis of the normal displacement as function of the tangential displacement and the tangential force respectively. By way of comparison with experimental observation, Fig. 23 shows similar behaviour measured for paper on steel [65]. 
Fig. 22 a The imposed periodic motion applied to the main mass. b The pre-sliding hysteresis of friction force as function of tangential displacement. c The lift-up butterfly curve of normal displacement as function of tangential displacement. d The lift-up butterfly curve of normal displacement as function of tangential force

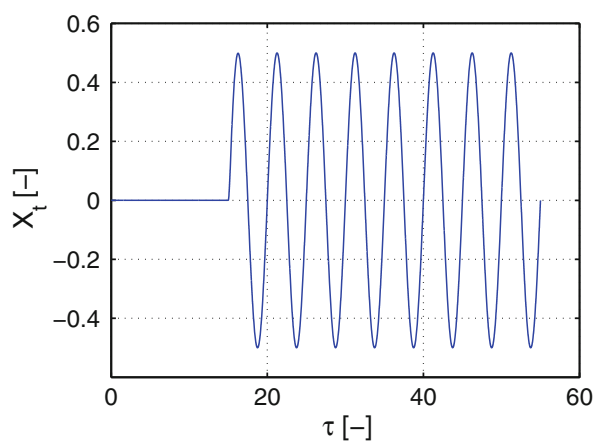

(a)

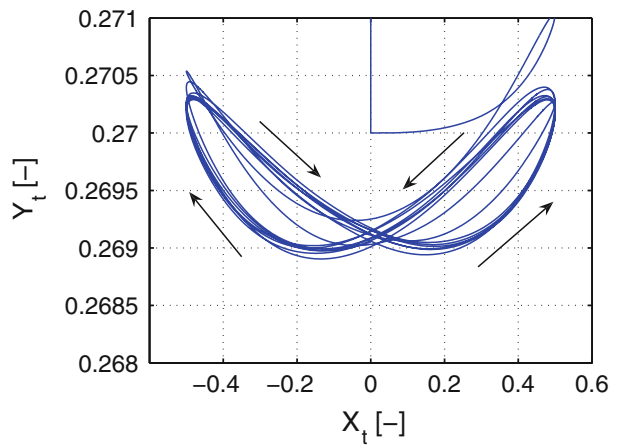

(c)

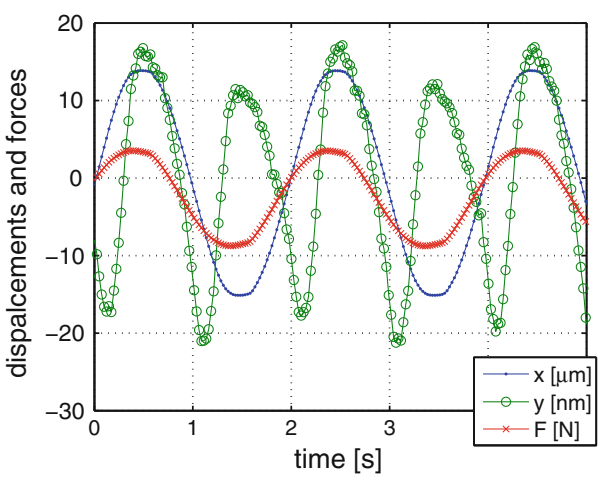

(a)

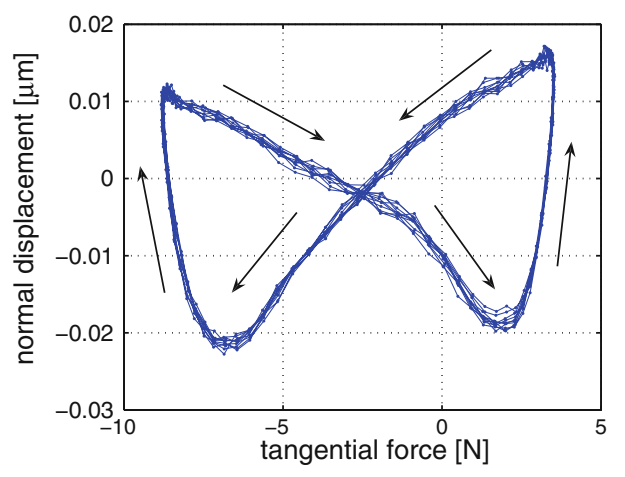

(c)

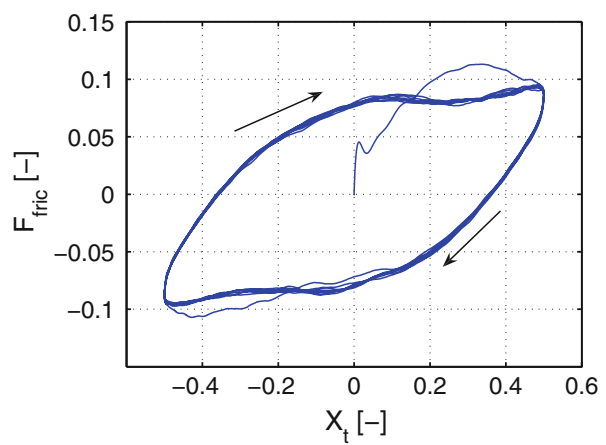

(b)

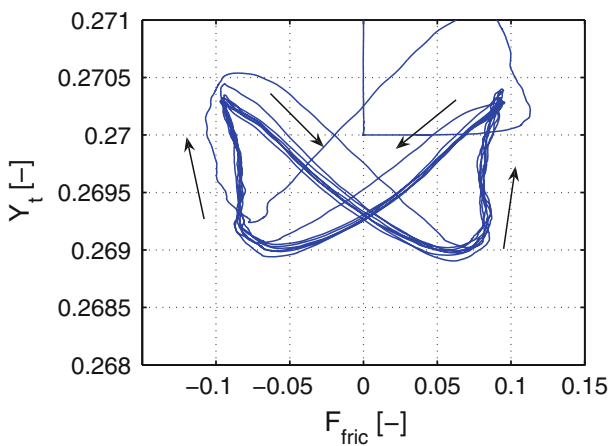

(d)
Fig. 23 a With the imposed periodic motion of a dry slider are associated periodic tangential (friction) force and normal (lift-up) displacement. b Depicts the tangential force against the tangential displacement, yielding the now well-known pre-sliding hysteresis curve (arrows indicate the direction of motion). c Shows the lift-up against the tangential force yielding the friction-lift butterfly, and $\mathbf{d}$ the displacement butterfly. Plots b, $\mathbf{c}$ and $\mathbf{d}$ are independent of the motion frequency in the test range $0.02-2.0 \mathrm{~Hz}$

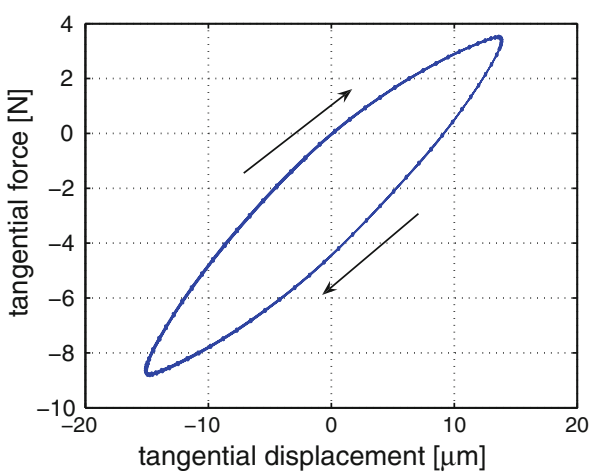

(b)

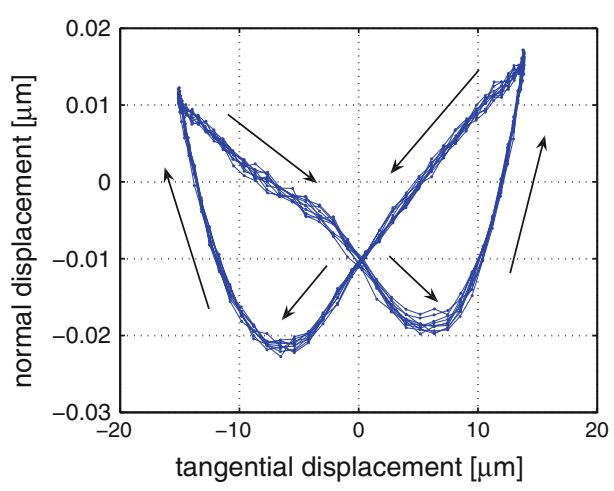

(d) 


\subsection{Stick-Slip Oscillatory Behaviour}

The generic model has the additional possibility of simulating stick-slip behaviour. Instead of imposing a displacement $X_{t}$ to the main mass, a (complex) spring is connected to the mass, where the displacement of the other end of the spring, $X_{m}$, is imposed. The stick and slip phases are clearly distinguishable in Fig. 24, depicting the position $X_{m}$, which is the desired position of the main mass, and the effective position $X_{t}$ of the main mass. This effect is also visible in the forces in the system in Fig. 25, where the spring force and the friction force in the contact are depicted. From these figures, one can follow the behaviour of the system. First, the main mass sticks to the counter profile, until the friction force equals the break away force. The mass starts to slide and the increasing velocity results in a decreasing friction force. Simultaneously, the spring force decreases because $\dot{X}_{m}$ is smaller than $\dot{X}_{t}$. The main

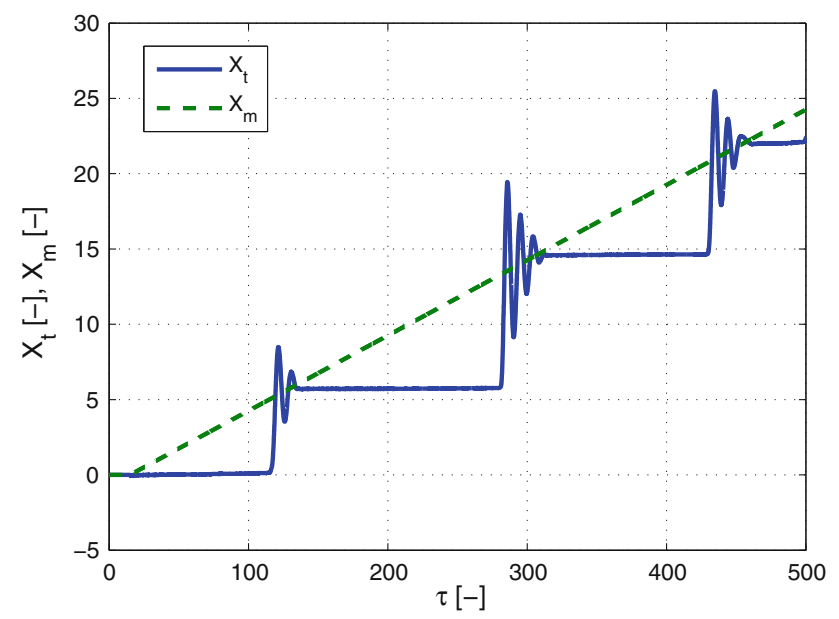

Fig. 24 Stick-slip behaviour. The desired position (dashed line) and the real position (solid line) as function of time mass accelerates until the force equilibrium is reached, resulting in a deceleration of the mass. Consequently, the friction force increases until the mass sticks again. The stick period starts with a damped oscillation, determined by the mass of the slider, the contact stiffness and the main stiffness, while the damping originates from the energy loss in the contact and the damping in the main spring. Figure 25 shows an inset with a detail of this oscillatory behaviour of the friction force and the spring force at the onset of sticking. Afterwards, the cycle restarts again. Similar behaviour was noticed by another modelling approach [25, 67], as well as, in another experimental study [53].

\subsection{On the Model Nature and Parameter Selection}

From the foregoing results, it is amply clear that model is able to qualitatively simulate known contact and friction dynamics using an arbitrary set of process parameters. However, a word about quantitative correspondence of the model, and the selection of realistic parameters to achieve this end, is in order here.

The model, as it stands now, is a heuristic synthesis of possible physical mechanisms, with their constituent parameters, involved in the friction phenomenon, which (i) are not exhaustive, and (ii) may not correspond in a direct, recognisable way to actual surface and material properties. Those two points have to be addressed, if we wish to make the model predictive rather than heuristic. Two ways may be pursued.

The first way is to continue treating the model as a generic one, with unknown parameters to be experimentally identified. In order to achieve this, we need to (i) carry out a sufficient number of friction experiments, covering the ranges of speeds and loads of interest for a given contact; (ii) (pre)select reasonable stochastic-distribution
Fig. 25 The applied spring force (dashed line) to the mass and the friction force (solid line) as function of time. The inset shows a detail of the oscillatory behaviour of the friction force and the spring force at the onset of sticking

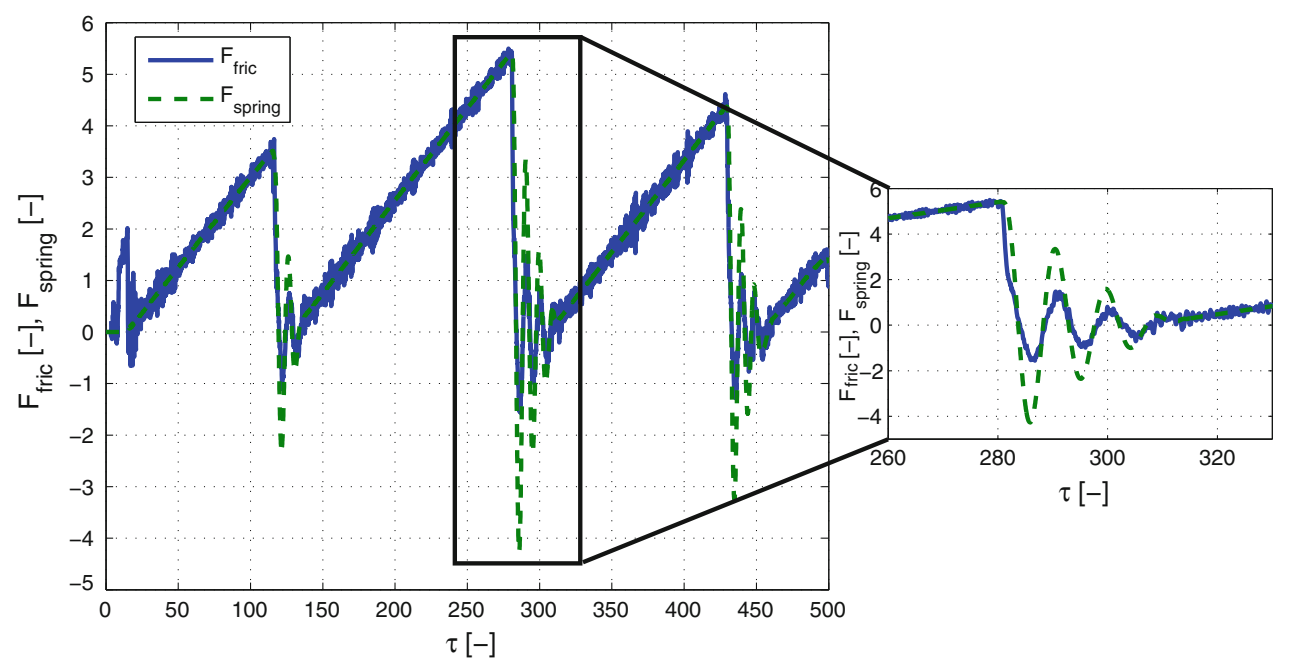


functions of the unknown parameters, and (iii) perform parameter identification by (optimally) fitting the experimental data to the model. In this case, we need not concern ourselves even with meaning or the orders-of-magnitude of the identified parameters, as long as we obtain a good fit. (For a discussion of the influence of some of parameters on the resulting friction behaviour, the reader is referred to [26]). Neither can we, in this case, indeed expect a correspondence of identified parameters with the actual properties of the surface (assuming that we are able to quantify those and convert them to model parameters), although this last possibility cannot be ruled out. This method has been applied with success in [67].

The second way is to make the model predictive. In this case, we must be able to translate the actual contact properties into model parameters. This is not a straightforward task, but the hurdles are not insurmountable, we believe. What we basically need to do is to convert the geometrical and material properties of the contacting surfaces into model parameters. The greatest hurdle here is interpreting the geometry of a surface, since the physical (mass, elasticity, etc.) properties will follow relatively easily once the form is known, e.g. by using structural-dynamics techniques. Regarding the geometry, or surface topography, much will depend on our model or conception of the surface. Firstly, if the surface is assumed to have a stochastic roughness distribution, we will need to include all the wavelengths present. This multi-scale aspect can lead to computationally intensive formulation requiring supercomputers. Secondly, if the surface topography is assumed to be fractal in nature, we need to construct a hierarchal surface model, of smaller asperities on top of larger ones in different levels, and then solving the problem to find the behaviour at each asperity level. By appropriately assembling the results, the global behaviour may be obtained.

\section{Discussion and Conclusions}

In this article, a newly developed generic model is presented and used to study the frictional behaviour of sliding contacts. In this model, the contact between two rubbing surfaces is reduced to the contact of a set of reduced asperities (each being represented by a mass connected to a normal and a tangential spring with nonlinear behaviour) and an elastic non-dissipative counter profile. The elasticplastic behaviour of each asperity is based on a two-element Maxwell-Slip model. Besides the contact force between the asperities and the counter profile, an adhesion law is imposed on the asperity mass when the asperity is in contact with the counter profile.

Despite its simplifications of a rough sliding contact, this model can simulate qualitatively the generally well-established friction force statics and dynamics observed in macroscopic experiments by many researchers. Furthermore, this model makes it possible to study the influence and relevance of the numerous parameters involved in the model and to relate these parameters to macroscopically observable and measurable quantities.

As future study, the relation between realistic surface and material parameters will be sought, which can yield a better understanding of the phenomena occurring in the contact. We believe that a dedicated modelling strategy, such one that is based on fractal analysis, can result in the accurate characterisation of real surfaces.

As a second future objective, we envisage the extension of the model with evolutionary surface properties, originating from wear processes taking place at the micro scale. Such an approach can help to improve the design of surfaces w.r.t. the life span optimisation of sliding contacts.

Acknowledgements This research is supported by the Fund for Scientific Research-Flanders (F.W.O.) under Grant FWO4283. This research is conducted utilising high performance computational resources provided by the University of Leuven, http://ludit. kuleuven.be/hpc. The scientific responsibility is assumed by its authors.

\section{Appendix}

See Table 1.

Table 1 Overview of the used model parameters

\begin{tabular}{llll}
\hline Parameter & Distribution & Distribution parameters & Value \\
\hline Asperties & & & \\
$\omega_{\mathrm{nT} i \text {,elastic }} / \omega_{\mathrm{N}}$ & Uniform & Min-max & $25-75$ \\
$\omega_{\mathrm{nT} i \text {, plastic }} / \omega_{\mathrm{N}}$ & Uniform & Min-max & $50-150$ \\
$\sigma_{\mathrm{T} i}$ & Uniform & Min-max & $0-20$ \\
$\omega_{\mathrm{nN} i, \text { elastic }} / \omega_{\mathrm{N}}$ & Uniform & Min-max & $10-30$ \\
$\omega_{\mathrm{nN} i \text { plastic }} / \omega_{\mathrm{N}}$ & Uniform & Min-max & $20-60$ \\
$\sigma_{\mathrm{N} i}$ & Uniform & Min-max & $0-30$ \\
$m_{i} / M$ & Uniform & Min-max & $0-2 \mathrm{e}-6$ \\
$\mathrm{~d} X_{\mathrm{T}, i}$ & Uniform & Min-max & $-0.25-0.25$ \\
$L i$ & Gaussian & Standard deviation & 0.11 \\
$\left|F_{\mathrm{adh}, i}\right|$ & Uniform & Min-max & $0-200$ \\
Profile & & & \\
$A$ & Constant value & - & $4 \mathrm{e}-3$ \\
$R$ & Uniform & Min-max & $0.02-0.1$ \\
$\omega_{\mathrm{nP} i} / \omega_{\mathrm{N}}$ & Uniform & Min-max & $0-1000$ \\
Stick-slip & & & \\
$\omega_{\mathrm{nT}} / \omega_{\mathrm{N}}$ & Constant value & - \\
$\zeta$ & Constant value & - & 0.75 \\
& &
\end{tabular}

${ }^{a}$ The mean value of the dimensionless asperity length $L_{i}$ is an arbitrary value, since only the relative displacement of the main mass w.r.t. its initial value is of interest 


\section{References}

1. da Vinci, L.: Codex Atlanticus

2. Amontons, G.: On the resistance originating in machines. In: Proceedings of the French Royal Academy of Sciences, pp. 206222 (1699)

3. Euler, L.: Sur le frottement des corps solides. Memoires de l'academie des sciences de Berlin 4, 122-132 (1748)

4. Coulomb, C.A.: Theorie des machines simples, en ayant egard au frottement de leurs parties, et a la roideur des cordages. In: Memoire de Mathematique et de Physics de l'Academie Royal, pp. 161-342 (1785)

5. Bowden, F.P., Tabor, D.: The Friction and Lubrication of Solids. Clarendon Press, Oxford (1950)

6. Popov, V.L., Psakhie, S.G.: Numerical simulation methods in tribology. Tribol. Int. 40, 916-823 (2007)

7. Berger, E.J.: Friction modeling for dynamic system simulation. Appl. Mech. Rev. 55(6), 535-577 (2002)

8. Perry, S.S., Tysoe, W.T.: Frontiers of fundamental tribological research. Tribol. Lett. 19(3), 151-161 (2005)

9. Persson, B.N.J.: Sliding friction. Surf. Sci. Rep. 33(3), 83-120 (1999)

10. Persson, B.N.J., Albohr, O., Tartaglino, U., Volokitin, A.I., Tosatti, E.: On the nature of surface roughness with application to contact mechanics, sealing, rubber friction and adhesion. J. Phys. Condens. Matter 17, 1-62 (2005)

11. Abbott, E.J., Firestone, F.A.: Specifying surface quality: a method based on accurate measurement and comparison. Mech. Eng. 55, 569-572 (1933)

12. Kragelsky, I.V.: Friction and Wear. Butterworths, Washington (1965)

13. Greenwood, J.A., Williamson, J.B.P.: Contact of nominally flat surfaces. Proc. R. Soc. Lond. A 295, 300-319 (1966)

14. Whitehouse, D.J. , Archard, J.F.: The properties of random surfaces on significance in their contact. Proc. R. Soc. Lond. A 316, 97-121 (1970)

15. Ogilvy, J.A.: Numerical simulation of friction between contacting rough surfaces. J. Phys. D Appl. Phys. 24(11), 2098-2109 (1991)

16. Hertz, H.: Über die Berührung fester elastischer Körper. Journal für die reine und angewandte Mathematik, 92 156-171 (1882)

17. Ford, I.J.: Roughness effect on friction for multi-asperity contact between surfaces. J. Phys. D Appl. Phys. 26, 2219-2225 (1993)

18. Tabor, D.: The Properties of Diamond, Chap. 10, pp. 325-350. Academic, London (1979)

19. Chang, W.R., Etsion, I., Bogy, D.B.: An elastic-plastic model for the contact of rough surfaces. Trans. ASME J. Tribol. 109, 257263 (1987)

20. Derjaguin, B.V., Muller, V.M., Toporov, Y.V.: Effect of contact deformations on the adhesion of particles. J. Colloid Interface Sci. 53(2), 314-326 (1975)

21. Tworzydlo, W.W., Cecot, W., Oden, J.T., Yew, C.H.: Computational micro- and macroscopic models of contact and friction: formulation, approach and applications. Wear 220(2), 113-140 (1998)

22. Karpenko, Y.A., Akay, A.: A numerical model of friction between rough surfaces. Tribol. Int. 34, 531-545 (2001)

23. Jackson, R.L., Green, I.: A finite element study of elasto-plastic hemispherical contact. ASME J. Tribol. 127(2), 343-354 (2005)

24. Quicksall, J.J., Jackson, R.L., Green, I.: Elasto-plastic hemispherical contact models for various mechanical properties. IMechE J. Eng. Tribol. 218(4), 313-322 (2004)

25. Lampaert, V.: Modelling and control of dry sliding friction in mechanical systems. PhD thesis, Katholieke Universiteit Leuven, Division of Production Engineering, Machine Design and Automation, Leuven, Belgium (2003)
26. Al-Bender, F., Lampaert, V., Swevers, J.: A novel generic model at asperity level for dry friction force dynamics. Tribol. Lett. 16(1), 81-93 (2004)

27. Geike, T., Popov, V.L.: Reduced description of mixed lubrication. Tribol. Int. 41, 542-548 (2008)

28. Björklund, S.: A random model for micro-slip between nominally flat surfaces. Trans. ASME J. Tribol. 119(4), 726-732 (1997)

29. Andrews, S., Sehitoglu, H.: A computer model for crack growth from rough surfaces. Int. J. Fatigue 22, 619-630 (2000)

30. Johnson, K.L., Kendall, K., Roberts, A.D.: Surface energy and the contact of elastic solids. Proc. R. Soc. Lond. A Math. Phys. Sci. 324, 301-313 (1971)

31. Tabor, D.: Surface forces and surface interactions. J. Colloid Interface Sci. 58, 1-13 (1976)

32. Muller, V.M., Yushenko, V.S., Derjaguin, B.V.: On the influence of molecular forces on the deformation of an elastic sphere and its sticking to a rigid plane. J. Colloid Interface Sci. 77, 91-101 (1980)

33. Maugis, D.: Adhesion of spheres: the JKR-DMT transition using a dugdale model. J. Colloid Interface Sci. 150(1), 243-269 (1992)

34. Vu-Quoc, L., Zhang, X., Lesburg, L.: Normal and tangential force-displacement relations for frictional elastic-plastic contact of spheres. Int. J. Solids Struct. 38, 6455-6489 (2001)

35. Ludema, K.C., Tabor, D.: The friction and visco-elastic properties of polymeric solids. Wear 9, 329-348 (1966)

36. Czichos, H.: Tribology a Systems Approach to the Science and Technology of Friction, Lubrication and Wear, vol. 1. Elsevier, Amsterdam (1978)

37. Iwan, W.D.: A distributed-element model for hysteresis and its steady-state dynamic response. J. Appl. Mech. 33(4), 893-900 (1966)

38. Goldfarb, M., Celanovic, N.: A lumped parameter electromechanical model for describing the nonlinear behavior of piezoelectric actuators. Trans. ASME J. Dyn. Syst. Meas. Control 119, 478-485 (1997)

39. Rizos, D.D., Fassois, S.D.: Presliding friction identification based upon the Maxwell Slip model structure. Chaos 14(2), 431-445 (2004)

40. Buckingham, E.: On physically similar systems: illustrations of the use of dimensional equations. Phys. Rev. IV(4), 345-376 (1914)

41. Bornemann, P.B., Galvanetto, U., Crisfield, M.A.: Some remarks on the numerical time integration of non-linear dynamical systems. J. Sound Vib. 252(5), 935-944 (2002)

42. Brockley, C.A., Davis, H.R.: The time-dependence of static friction. Trans. ASME J. Lubr. Technol. 90, 35-41 (1968)

43. Rabinowicz, E.: Friction and Wear of Materials. Wiley, New York (1965)

44. Mulhearn, T.O., Tabor, D.: Creep and hardness of metals: a physical study. J. Inst. Met. 89(1), 7-12 (1960)

45. Bowden, F.P., Tabor, D.: The Friction and Lubrication of Solids, Part II. Clarendon Press, Oxford (1964)

46. Gitis, N.V., Volpe, L.: Nature of static friction time dependence. J. Phys. D Appl. Phys. 25(4), 605-612 (1992)

47. Berthoud, P., Baumberger, T.: Role of asperity creep in time- and velocity-dependent friction of a polymer glass. Europhys. Lett. 41(6), 617-622 (1998)

48. Baumberger, T.: Contact dynamics and friction at a solid-solid interface: material versus statistical aspects. Solid State Commun. 102(2-3), 175-185 (1997)

49. Dowson, D.: History of Tribology. Longman, London (1979)

50. Baumberger, T.: Physics of Sliding Friction, Chap. 1. Dry Friction Dynamics at Low Velocities, pp. 1-16. Kluwer Academic Publishers, Dordrecht (1996)

51. Heslot, F., Baumberger, T., Perrin, B., Caroli, B., Caroli, C.: Creep, stick-slip, and dry-friction dynamics: experiments and a heuristic model. Phys. Rev. E 49(6), 4973 (1994) 
52. Dieterich, J.H., Kilgore, B.D.: Direct observation of frictional contacts: new insights for state-dependent properties. Pure Appl. Geophys. 143, 283-302 (1994)

53. Lampaert, V., Al-Bender, F., Swevers, J.: Experimental characterisation of dry friction at low velocities on a developed tribometer setup for macroscopic measurements. Tribol. Lett. 16(1-2), 95-105 (2004)

54. Mayergoyz, I.: Mathematical Models of Hysteresis. SpringerVerlag, New-York (1991)

55. Al-Bender, F., Symens, W.: Dynamic characterization of hysteresis elements in mechanical systems, Part 1. Theoretical analysis. Chaos Interdiscip. J. Nonlinear Sci. 15(1), 1-11 (2005)

56. Masing, G. (1926) Eigenspannungen und Verfestigung beim Messing. In: Proceedings of the 2nd International Congress on Applied Mechanics. Zürich, Swiss, pp. 332-335

57. Smith, K.N., Watson, P., Topper, T.H.: Stress-strain function for fatigue of metals. J. Mater. 5(4), 767-778 (1970)

58. Nouri, B.M.Y.: Friction identification in mechatronic systems. ISA Trans. 43, 205-216 (2004)

59. Kappagantu, R.V., Feeny, B.F.: Proper orthogonal modes of a beam with frictional excitation. In: Guran, A. (ed.) Proceedings of the First International Symposium on Impact and Friction of Solids, Structures and Intelligent Machines, vol. 14 of B, pp. 167-172 (1998)

60. Yanada, H., Sekikawa, Y.: Modeling of dynamic behaviors of friction. Mechatronics, 18, 330-339 (2008)
61. Al-Bender, F., De Moerlooze, K.: On the relationship between normal load and friction force in pre-sliding frictional contacts, Part 1: Theoretical analysis. Submitted to Wear (2009). doi: 10.1016/j.wear.2010.02.010

62. De Moerlooze, K., Al-Bender, F.: On the relationship between normal load and friction force in pre-sliding frictional contacts, Part 2: Experimental investigation. Submitted to Wear (2009). doi:10.1016/j.wear.2010.02.008

63. Hess, D.P., Soom, A.: Friction at a lubricated line contact operating at oscillating sliding velocities. J. Tribol. 112, 147-152 (1990)

64. Harnoy, A., Friedland, B., Rachoor, H.: Modeling and simulation of elastic and friction forces in lubricated bearings for precise motion control. Wear 172, 155-165 (1994)

65. Al-Bender, F., De Moerlooze, K., Vanherck, P.: Lift-up butterflies in friction. Manuscript in preparation (2010)

66. Tolstoi, D.M.: Significance of the normal degree of freedom and the natural normal vibrations in contact friction. Wear 10(3), 199-213 (1967)

67. Al-Bender, F., Lampaert, V., Swevers, J.: Modeling of dry sliding friction dynamics: from heuristic models to physically motivated models and back. Chaos Interdiscip. J. Nonlinear Sci. 14(2), 446460 (2004) 